\title{
Linking electronic and molecular structure: insight into aqueous chloride solvation $†$
}

Cite this: Phys. Chem. Chem. Phys., 2013, 15, 13169

\author{
Ling $\mathrm{Ge}^{* a}$ Leonardo Bernasconi $^{\mathrm{b}}$ and Patricia Hunt ${ }^{\star a}$
}

\begin{abstract}
Aqueous chloride solutions are ubiquitous and diverse; systems include sea water, atmospheric droplets, geological processes and biological organisms. However, despite considerable effort, a complete microscopic model of the hydration shell, and local electronic structure of the aqueous chloride ion and its dynamics has not been established. In this work we employ ab initio molecular dynamics to study an aqueous chloride solution. In particular, local solvation events and the electronic structure around the chloride ion are interrogated. We employ the Effective Molecular Orbital (EMO) method which partitions the electronic structure into solute and solvent components while maintaining a rigorous quantum mechanical description of both. Movement of the chloride highest occupied molecular orbital (HOMO) energy within the valence band of water is revealed. The chloride ion has little impact on the average water electronic structure, however, locally the electronic effect of the chloride ion is significant. With the Hofmeister series in mind we find that the electronic effect of the chloride ion extends beyond the first solvation shell, but not beyond the edge of the second solvation shell. The chloride ion sits near the centre of the Hofmeister series because of an essential degeneracy between water-water and water- $\mathrm{Cl} \mathrm{H-bonding} \mathrm{and} \mathrm{because} \mathrm{of} \mathrm{a} \mathrm{strong} \mathrm{similarity} \mathrm{in} \mathrm{the} \mathrm{water} \mathrm{and} \mathrm{chloride} \mathrm{elec-}$ tronic structure. The chloride ion prefers to be symmetrically solvated by six $\mathrm{H}$-bonding water molecules, however, the chloride HOMO energy and the coordination number oscillate in response to local fluctuations driven by the dynamics of the bulk water. A combined structural and electronic analysis has led to a distinction between two types of water molecule within the first solvation shell, those that $\mathrm{H}$-bond to the chloride ion, and those that remain local (i.e. within the first solvation shell) but which $\mathrm{H}$-bond to other water molecules. There are indications that these exhibit different dynamics with respect to residence times and rotational vs. translational motion.
\end{abstract}

Received 13th February 2013, Accepted 29th May 2013

DOI: $10.1039 / \mathrm{c} 3 \mathrm{cp} 50652 \mathrm{e}$

www.rsc.org/pccp
$\mathrm{Cl}$ anions can enter the atmosphere from the sea surface. Airborne aqueous sea-salt aerosols are a global source of molecular chlorine in the troposphere. ${ }^{1-3}$ Once in the atmosphere, $\mathrm{Cl}$ anions impact on the ozone cycle. ${ }^{4}$ They also act as nucleation sites for aerosols which perturb the radiation balance of the earth. ${ }^{5}$ A significant source of uncertainty in the current understanding of global climate change is atmospheric aerosol radiative forcing. ${ }^{6}$

Natural water sources contain $\mathrm{Cl}$ anions which play an important role in oceanographic and geological processes. For example, small variations in sea surface salinity can have dramatic effects on the water cycle and ocean circulation. ${ }^{7}$ Changing environmental conditions can alter processes such as the diffusive transport of saltwater into freshwater sediments. This in turn effects the present and historical character of sediments in lakes and lagoons. ${ }^{8}$ With reducing fresh water sources, the processing of saline water has become of increasing importance. The models used to understand $\mathrm{Cl}$ anion solute 
transport require knowledge of the hydration environment and reactivity of the $\mathrm{Cl}$ anion in aqueous solution.

In biological systems, the ability of salts to induce or retard the precipitation of proteins and non-polar (hydrocarbon) solutes from an aqueous solution is important, and follows a trend known as the Hofmeister series. ${ }^{9-13}$ Similar trends are found for surface tension, chromatographic selectivity, protein denaturation temperatures, protein stability, protein-protein interactions, protein crystallisation, enzyme activity, and colloid stability. ${ }^{9,10,13}$ Properties such as the Jones-Dole B coefficient of viscosity, the permeation of ions through membranes, measurements on $\mathrm{pH}$ buffers, zeta and membrane potentials, colloidal force measurements, ion pumps, neutron scattering and direct force measurements are all interpreted in terms of classical models including ion (Hofmeister) and hydration sphere effects. ${ }^{9,14,15}$

Traditionally, the Hofmeister series has been related to the effect of an ion on the (H-bonding) structure of liquid water. Structure makers (kosmotropes) decrease the solubility of nonpolar molecules ("salting out"). Structure breakers (chaotropes) enhance the solubility of non-polar molecules ("salting in"). ${ }^{16}$ The increased solubility of non-polar molecules is due to a reduction in the negative entropy (or enhanced disorder) in the surrounding solvent, structure breakers facilitate this process. Structure makers are assumed to enhance the tetrahedral network of water making it more icelike.

Small anions with a higher charge density have a tight first solvation shell, they form $\mathrm{H}$-bonds to local water molecules and are structure makers. Small ions are also repelled from an airwater interface. Larger but more diffuse anions without a strong solvation shell are structure breakers. Large ions tend to gather at the air-water interface. This study focuses on the $\mathrm{Cl}$ anion, which sits near the middle of the Hofmeister series for anions: $\mathrm{SO}_{4}{ }^{2-}>\mathrm{F}^{-}>\mathrm{CH}_{3} \mathrm{COO}^{-}>\mathrm{Cl}^{-}>\mathrm{Br}^{-}>\mathrm{I}^{-}>\mathrm{SCN}^{-}$. The $\mathrm{Cl}$ anion is generally considered a weak structure breaker, ${ }^{10,14}$ and is known to be neutral with respect to a propensity to collect at the water-air boundary. ${ }^{17,18}$ The $\mathrm{Cl}$ anion is small enough to be hydrated, but it is also large enough to be polarizable.

Experimental and theoretical results from the literature (outlined shortly) reveal a rather inhomogeneous picture of the hydrated chloride ion solvation shell structure. Moreover, there is little information about the underlying electronic structure which drives the dynamics associated with solvation. In this paper, we link the electronic and molecular structure and provide insight into aqueous chloride solvation.

The effect of aqueous solvated ions beyond the first or the second solvation shell is less clear, particularly as this applies to H-bonding between water molecules, and this topic has generated significant debate in the recent literature. ${ }^{13,16,19}$ There is physical and simulation based evidence for both the traditional view of long range ion effects, ${ }^{14,20-24}$ and a more recent proposal that ion effects are purely local. ${ }^{15,25-33}$ There is also evidence that hydration effects beyond the first solvation shell are dependent on the particular cation-anion combination and on the concentration..$^{20,27,28,34}$ Moreover, some studies show effects, which are minor at a long range but significant at a short range. ${ }^{20,35}$
It has also been suggested that the effects do not relate to the structuring of water, but that ions interact directly with the protein. ${ }^{13,36}$ How a tight solvation sphere enhances the long range structure in water, or how a large polarizable anion disrupts the long range structure in water is significantly more tenuous and not easy to rationalize, particularly in light of the dynamic nature of liquid water. It is a significant issue that the Hofmeister series cannot be rationalised by reference to a molecular level picture. ${ }^{24,37}$ It has also been suggested that the terms "structure maker" and "structure breaker" are not supported at the molecular scale. ${ }^{20}$

Simulations have indicated that fixed charge models are not sufficient to describe ions in aqueous solution, and the ability to induce a dipole centered on the ion is important. ${ }^{16,17}$ However, it has also been found that Hofmeister related phenomena can also be recovered via other routes, such as increasing the size of the anion, using optimised non-polarizable force fields, including electrostatic damping, or by considering the air/water interfacial shape. ${ }^{38-41}$

Thus, there is clear motivation for investigating the solvation of small ions, and the chloride ion in particular. It is important to understand solvation at the molecular level because this information impacts on our understanding and modelling of atmospheric, land and sea based water bodies, geological processes and biochemical systems. These molecular scale phenomena are ultimately linked to key current global challenges; climate change prediction and clean water generation.

A variety of experimental methods have been used to investigate the hydration structure of chloride ions in water, these include neutron and X-ray diffraction techniques, ${ }^{20,21,23,35,42,43}$ X-ray absorption spectroscopy (EXAFS and XANES), ${ }^{44}$ gel sieving chromatography, ${ }^{45}$ Raman and IR spectroscopy, ${ }^{30,46}$ mass spectroscopy $^{47,48}$ and photoelectron spectroscopy. ${ }^{49}$ More recently the development of new spectroscopic methods, femtosecond infrared pump-probe IR spectroscopy and terahertz dielectric relaxation spectroscopy, has provided additional details. ${ }^{10,25,50-52}$ The optical Kerr effect and dielectric relaxation spectroscopy have also offered insight. ${ }^{53}$ However, experimentally derived coordination numbers are significantly scattered, ranging from 3 to $8 .^{20,21,44,46,49,54}$ Thus the number of water molecules surrounding the chloride ion in the first solvation shell is still unclear.

A range of theoretical methods, from molecular dynamics (MD) simulations through $a b$ initio $\mathrm{MD}$ and full quantum chemical calculations, have been applied to the water-chloride interaction and $\mathrm{Cl}$ anion solvation. Difficulties in determining the structure of the solvation environment from MD studies arise because of the sensitivity of results to the details of water$\mathrm{Cl}$ and water-water interaction potentials. ${ }^{17,55-60}$ Differently parametrized potentials, with and without a treatment of molecular polarizability, give different structural properties. $^{24,61,62}$ For example, simulations of small hydrated clusters have demonstrated that changing the polarizability of the "chloride" ion determines whether the whole cluster is symmetric or polarised. ${ }^{63}$ In contrast, simulations of larger clusters 
(20-255 water molecules) have indicated that the water (and not ions) polarisability is important. ${ }^{61,64}$ Non-polarizable potentials tend to predict an interior location for the chloride anion, while polarizable models predict a surface location for the anion. ${ }^{65}$ More recently it has been suggested that the degree of polarisation induced in classical models may be too strong. ${ }^{66} \mathrm{Ab}$ initio MD naturally recovers polarisation for both the ion and water; studies have been carried out on aqueous chloride solutions, ${ }^{67-69}$ and chloride water clusters. ${ }^{65,70}$ In both cases an asymmetric solvation structure is found. Quantum mechanics/molecular mechanics simulations (employing a HF QM description) of a $\mathrm{Cl}$ anion in water have also been carried out. ${ }^{71}$ Uncertainty in the coordination number is evinced from theoretical studies, with variations between 5.1 and 8.4 depending on the simulation techniques adopted. ${ }^{56,60,67,68,71-77}$ Thus, despite extensive efforts, a complete microscopic model of the hydration shell of the aqueous chloride ion and its dynamics is still being debated.

Thus, the solvation of $\mathrm{Cl}$ anions in an aqueous environment has been the subject of a substantial amount of experimental and theoretical work. Nevertheless, despite concerted efforts to unravel the effect of ions on the structure, dynamics and electronic features of water, many questions remain open. How chloride ions are accommodated in the solvent hydrogen bonding network, and how they influence the solvent structure, is not well understood. Moreover, there is little information on the intimate connection between the electronic structure of the solute chloride ion and aqueous solvent.

In this work we employ $a b$ initio MD to study an aqueous chloride solution and in particular focus on the local solvation structure around the $\mathrm{Cl}$ anion. In contrast to previous ab initio studies, we concentrate on the nature of the electronic interactions between the solute $\mathrm{Cl}$ anion and the solvent, which is interpreted on the basis of the Effective Molecular Orbital (EMO) method. ${ }^{78}$ A combination of the EMO method and a more traditional structural analysis is then used to examine the relationship between the coordination environment and the electronic structure of the $\mathrm{Cl}$ anion. Moreover, we investigate the extent to which the $\mathrm{Cl}$ anion influences water molecules beyond the first solvation shell.

The paper is organised as follows. In Section 2 the computational details of the MD simulations are presented. In the Results and discussion (Section 3) radial distribution functions and coordination numbers are discussed and benchmarked. The results of the EMO analysis are presented, the extent of electronic coupling between the chloride ion and water molecules is analysed, the local solvation environment is interrogated, the dipole moments of the system reported and rare events which involve water molecules entering or leaving the first solvation shell are analysed. Results are summarised in the Conclusions (Section 4).

\section{Computational details}

$A b$ initio $\mathrm{MD}$ calculations of a $\mathrm{Cl}$ anion solvated in liquid water have been performed using the CPMD program. ${ }^{79,80}$ The plane wave-pseudopotential implementation of the Kohn-Sham method used in this code has been validated in previous studies of aqueous systems. ${ }^{78,81-83}$ Similar to the majority of these simulations the BLYP functional has been employed. ${ }^{84,85}$ Norm conserving pseudo potentials have been employed and expanded in a plane wave basis up to an energy cutoff of $70 \mathrm{Ry}$ at the $\Gamma$ point.

The system consists of 63 water molecules and one chloride ion in a simple cubic supercell of dimension $12.42 \AA$ corresponding to the experimental density under ambient conditions with chloride ion concentration $\left(0.88 \mathrm{~mol} \mathrm{~L}^{-1}\right)$. The starting configuration was obtained from an equilibrated classical MD simulation using an extended simple point charge (SPC/E) model for water, in combination with a chloride-water potential. ${ }^{60,86}$ The $a b$ initio trajectory was then equilibrated to an average temperature of $298 \mathrm{~K}$ using CPMD for $2.76 \mathrm{ps}$. A fictitious mass of 600 a.u. and a time step of 5 a.u. were used in the integration of the equations of motion. The simulation temperature was controlled through the equilibration phase via a Nosé-Hoover thermostat with a frequency of $1100 \mathrm{~cm}^{-1} \cdot{ }^{87} \mathrm{In}$ a real system the $\mathrm{Cl}$ anion will always be present with a neutralising cation, however it has been found experimentally that the $\mathrm{Cl}$ anion solvation structure is predominantly independent of the nature of the counter ion. ${ }^{20,21,35,46,52}$ In these simulations the counter ion has been replaced by a positive neutralising background charge. For the electronic analysis 80 structures were collected over the post-equilibration phase, of 28.8 ps at equal intervals of 0.36 ps.

The EMO method interprets the time evolution of the electronic and molecular structure of both solute and solvent. ${ }^{78}$ This analysis is based on a rigorous quantum mechanical description of both the solute and solvent, i.e. it does not rely on partitioning of the system into quantum-mechanical (solute) and classical (solvent) components. A brief description of the method can be found in the Fig. S1 and S2 (ESI $\dagger$ ). The EMO analysis determines an effective energy $\alpha$ for each EMO and $\beta$ which represents the electronic coupling between orbitals on different molecules. Thermal broadening can be obtained as the standard deviation in the energy of the EMOs, $\Delta \alpha$.

The EMO analysis has been successfully applied to pure liquid water. ${ }^{78}$ An isolated water molecule has $C_{2 \mathrm{v}}$ symmetry and valence MOs $2 a_{1}, 1 b_{1}, 3 a_{1}$ and $1 b_{2}$ (Fig. S3, ESI $\dagger$ ). A liquid environment is disordered and there is no symmetry, however, the EMOs strongly resemble the standard MOs for an isolated water molecule. ${ }^{78}$ This close resemblance is by no means predetermined and is a validation of the EMO method. For clarity, we will continue to use the isolated atomic or molecular symmetry labels in referring to individual atomic orbitals, MOs and bands in the aqueous system.

The EMO approach is sufficiently general to be used on gasphase systems, as well as condensed (periodic) systems, an illustrative example of the simple gas-phase $\left(\mathrm{Cl}^{-}+\mathrm{H}_{2} \mathrm{O}\right)$ cluster is provided in Fig S4 (ESI + ). Changing the functional from BLYP to PBE does not significantly affect the results (Table S1, Fig. S5 and S6, ESI $\dagger$ ).

BLYP is well known to be unable to match the absolute position of water orbital energy levels, and thus we apply an 
energy shift to align the computed water $1 b_{2}$ HOMO peak maxima to that obtained experimentally, $-11.16 \mathrm{eV} .{ }^{88} \mathrm{~A}$ comparison of orbital energies computed at the BLYP, B3LYP and MP2 levels for the simple gas-phase $\left(\mathrm{Cl}^{-}+\mathrm{H}_{2} \mathrm{O}\right)$ cluster is provided in Fig. S7 (ESI + ). Water can be thought of as an oxide with a band gap of $8.7 \mathrm{eV}$ (wide band gap insulator) which is underestimated by BLYP. Traditionally one might assume that the HOMO or valence band maximum (VBM) is correctly determined and that the error lies in the position of the LUMO or conduction band minimum (CBM). However, not only is the water CBM incorrectly referenced in BLYP but also the water VBM. Indications were already evident in 2005 that this was the case $^{89}$ but this has now been firmly established. ${ }^{90}$ The origin of this error is thought to be the self-interaction or delocalisation error. It has been shown that the band gaps of crystalline insulators computed at the DFT/GGA level are substantially affected by the amount of Hartree-Fock exchange included in the exchange-correlation functional, and that not only the CBM but also the VBM are influenced by this effect. ${ }^{91}$ More recently it has been shown that the VBM of an aqueous solution is displaced more (relative to the experimental value) than the CBM. ${ }^{90,92}$ Thus the electronic position of the water HOMOs from our BLYP simulations are approximately $3.5 \mathrm{eV}$ higher than they should be, and we rescale to the experimentally determined valence band, where the peak center is at $-11.16 \mathrm{eV}$. More positively, the lower orbitals appear to be at roughly the correct energies when referenced to the water conduction band. $^{88}$

In order to compare the magnitude of the water-chloride and water-water interaction energies, B3LYP and MP2 calculations employing a cc-pVTZ basis set have been carried out using Gaussian 09. ${ }^{93,94}$ All structures have been optimised under no symmetry constraints with enhanced convergence criteria for the energy and density, and are confirmed as minima by frequency calculations. A thermochemical analysis has been carried out to determine zero point energy (ZPE) corrections and an estimate of the basis set superposition error (BSSE) has been obtained using the counterpoise method. ${ }^{95}$

\section{Results and discussion}

\subsection{Benchmarking: comparison of RDFs with experiment and simulation}

When a $\mathrm{Cl}$ anion inserts into the H-bonding network of liquid water directional Cl-water $\mathrm{H}$-bonds are formed. The H-bonding interactions are not static; thermal and electronic motions allow H-bonds to break and reform in new patterns. The $\mathrm{Cl}$ anion can "mimic" a water molecule in undertaking at least two $\mathrm{H}$-bond acceptor interactions, however there is an absence of H-bond donor interactions.

Radial distribution functions (RDFs) provide an average $\mathrm{H}$-bond length and an indication of the variation in length for the water-water and $\mathrm{Cl}-$ water $\mathrm{H}$-bonds. They also allow us to evaluate the quality of the simulations by comparing computed RDFs with those obtained experimentally. Relevant data from our simulation are presented in Table 1.
Table 1 Position of peak maxima and minima in the RDF plots, $r$ in $\AA$, and coordination numbers (CN)

\begin{tabular}{lllll}
\hline & $r^{1 \text { st }}$ maxima & $r^{2 \text { nd }}$ maxima & $r^{1 \text { st }}$ minima & $\mathrm{CN}$ \\
\hline$g_{\mathrm{OO}}$ & 2.74 & 4.41 & 3.29 & 4.2 \\
$g_{\mathrm{OH}}$ & Bond & 1.76 & 1.23 & - \\
$g_{\mathrm{ClO}}$ & 3.15 & 4.74 & 3.83 & 6.3 \\
$g_{\mathrm{ClH}}$ & 2.19 & 3.62 & 2.90 & 5.1 \\
\hline
\end{tabular}

The RDFs of pure liquid water are typically well reproduced by CPMD simulations. ${ }^{81,83}$ The $\mathrm{O}-\mathrm{O}$ and $\mathrm{O}-\mathrm{H}$ RDFs for the chloride solution simulated here (Fig. S8 and S9, ESI $\dagger$ ) resemble those of pure water, indicating that the $\mathrm{Cl}$ anion does not significantly affect the long range water structure at this concentration. ${ }^{83}$ For comparison, the experimental $\mathrm{O}-\mathrm{O}$ and O-H RDF maxima for pure water are $2.88 \AA$ and $1.85 \AA$ respectively. The experimental first minimum in the $\mathrm{O}-\mathrm{O}$ RDF occurs at $3.3 \AA$ and the coordination number is $4.5 .^{96}$

The computed $\mathrm{Cl}-\mathrm{O}$ and $\mathrm{Cl}-\mathrm{H}$ RDFs $\left(\mathrm{Cl}: \mathrm{H}_{2} \mathrm{O}\right.$ ratio $\left.1: 63\right)$ are presented in Fig. 1. Relevant neutron diffraction experiments have been used to study solutions bracketing our $\mathrm{Cl}$ : water ratio: $1: 11$ and $1: 83$ with counter ions $\mathrm{Na}^{+}$and $\mathrm{K}^{+} .^{20,21}$ The experimental $g_{\mathrm{ClO}}$ and $g_{\mathrm{ClH}}$ RDF maxima bracket our results. Moreover, greater variation was found when changing the counter ion, than when changing the concentration. Other experiments have found the first peak of the $\mathrm{Cl}-\mathrm{O}$ RDF to be in the range of 3.01-3.36 $\AA$ varying slightly with concentration. ${ }^{44,56,71}$ The experimentally determined coordination numbers for aqueous chloride solutions vary with concentration; for ratios of $1: 11-1: 83\left(\mathrm{Cl}: \mathrm{H}_{2} \mathrm{O}\right)$ the $\mathrm{Cl}-\mathrm{O}$ and $\mathrm{Cl}-\mathrm{H}$ coordination numbers vary between 5.7-7.0 and 5.9-6.1 respectively. ${ }^{20}$ The computed $\mathrm{Cl}-\mathrm{H}$ coordination number is within the lower error bound $( \pm 1.1)$ of the experimental values. Comparison with the experimental results described above indicate that our simulation can be considered to be reflective of aqueous chloride solutions (around a concentration of $0.88 \mathrm{~mol} \mathrm{~L}^{-1}$ for $\mathrm{K}^{+}$and $\mathrm{Na}^{+}$monovalent counter ions).

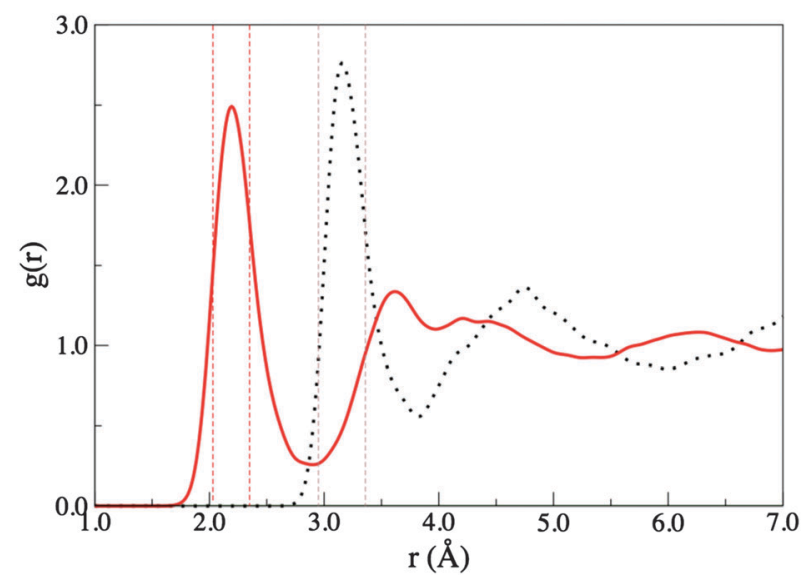

Fig. 1 Radial distribution functions for $\mathrm{Cl}-\mathrm{O}$ (dotted line) and $\mathrm{Cl}-\mathrm{H}$ (solid line) for an aqueous chloride solution. Dashed lines represent the range of the first maxima of the RDF from experiments. ${ }^{20,44,56,71}$ 


\subsection{Effective MO analysis}

A unique aspect of the EMO analysis is that it allows resolution of the local orbitals associated with each molecule, solute orbitals can be resolved from the sea of solvent orbitals, and it is possible to investigate relationships between the local solvation environment and the local coordination environment.

The EMOs of the $\mathrm{Cl}$ anion at one time step (19.32 ps) of the trajectory are plotted in Fig. 2. The effective MOs closely resemble the respective atomic and molecular orbitals of the isolated molecules with slight distortions due to the influence of neighbouring water molecules.

In a condensed environment the individual molecular orbitals form 'bands'. Fig. 3 shows the density of states (DOS) for liquid water with a single $\mathrm{Cl}$ anion impurity, computed by averaging over 80 instantaneous atomic configurations taken from an $a b$ initio trajectory of $\sim 30 \mathrm{ps}$ duration. The DOS of pure liquid water has been studied in detail previously using first-principles approaches and photoemission spectroscopy. ${ }^{78,88}$ The effects on the DOS of adding a $\mathrm{Cl}$ anion at this concentration appear to be relatively minor, and there are no large changes in position or intensity. However, the overlap of the $\mathrm{Cl} 3 \mathrm{p}$ and
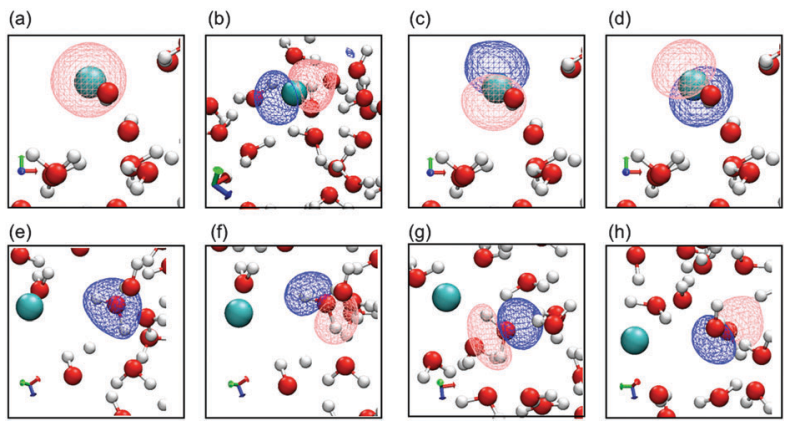

Fig. 2 EMO plots of the $\mathrm{Cl}$ anion and one water molecule in the first solvation shell. Cl solute EMOs (a) 3s-like orbital and (b)-(d) 3p-like orbitals, water EMOs (e) $2 a_{1}$, (f) $1 b_{1}$, (g) $3 a_{1}$ and (h) $1 b_{2}$

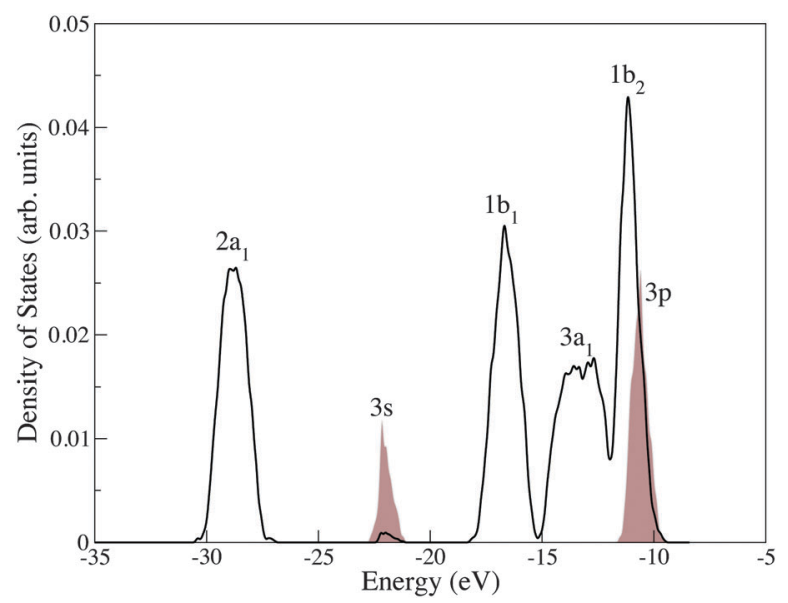

Fig. 3 Total density of occupied states for an aqueous chloride solution. The water bands are labeled following the convention for a $\mathrm{C}_{2 v}$ symmetry $\mathrm{H}_{2} \mathrm{O}$ molecule. The chloride ion peaks are represented by shaded areas which, for clarity, have been magnified by a factor 10 . water EMOs suggests a potentially significant level of orbital mixing.

The $\mathrm{Cl}$ anion can be thought of as a charged defect in a wide band gap oxide (water). The question arises of how accurately the $\mathrm{Cl}$ anion HOMO is positioned relative to the water VBM (given the problems BLYP has in positioning the water conduction band). Experimentally, the $\mathrm{Cl}$ anion detachment level is just above $(-8.8 \mathrm{eV})$ the valence band edge of water $(-9.9 \mathrm{eV})$ and in spectra the $\mathrm{Cl}$ anion $3 \mathrm{p}$ orbitals appear as a shoulder on the water valence band due to broadening. ${ }^{88,97}$ Solid state charge defect energies can be in error by amounts as large as $1 \mathrm{eV}$, and similar errors can occur in aqueous solution. ${ }^{92}$ This is significant as it can determine whether the $\mathrm{Cl}$ anion HOMO lies above or is buried within the water band, changing the character of the HOMO for the whole system. Broadening of the $\mathrm{Cl}$ anion HOMO (thermal and electronic) complicates the matter further, and thus the $\mathrm{Cl}$ anion HOMO is mixed up with the valence band of water. The extent to which the delocalisation error in BLYP affects this mixing is not known. Our results indicate that when the valence band of water is adjusted to match the experimental value, the $\mathrm{Cl}$ anion HOMO peak center occurs at $-10.12 \mathrm{eV}$, which lies above the band maxima, but on the band edge of water. Previous calculations treating the ion at the MP2 level but the surrounding water molecules as point charges obtained $1.04 \mathrm{eV}$ compared to an experimental estimate of $0.6 \mathrm{eV}$, we obtain a maximum broadening of $1.39 \mathrm{eV}{ }^{88,97} \mathrm{~A}$ further application of the EMO method to a simulation in which a hybrid exchange functional has been employed, and a comparison with the results presented here would be highly insightful.

Over the course of a trajectory the electronic structure of the solute and solvent molecules varies in time. Unique to the EMO method is the ability to monitor variations of the individual effective MOs over time. Fig. 4 shows the position of the $\mathrm{Cl}$ anion effective HOMO relative to the water $1 b_{2}$ HOMO band for selected points along a trajectory.

Over the course of the trajectory, three parameters $\alpha, \Delta \alpha$ and $\beta$ have been computed and averaged for the water and $\mathrm{Cl}$ anion EMOs, Table 2. $\alpha$ is the center of each effective MO band. For example, $\alpha$ for the $1 b_{2}$ effective MO relates to the blue line at the center of the water HOMO band in Fig. 4, and it is the average of these values over the whole trajectory. $\Delta \alpha$ is the "thermal broadening" of a band due to changes in the molecular environment over time, and is related to temperature fluctuations (evident in the oscillations of the band edges). $\beta$ is the electronic broadening due to overlap with orbitals from other molecules. The combined effect of the thermal and electronic broadening contribute to the total "width" of a band. Details of how these quantities are derived can be found in ref. 78 .

From Fig. 4 it is evident that while individual water EMOs vary significantly in energy (see the top and bottom of the water HOMO band), the average energy (band center) remains stable over the whole trajectory. The system HOMO is the "reactive" region and in the EMO analysis the HOMO can be unambiguously associated with a particular molecular species. The $\mathrm{Cl}$ anion dominates the system HOMO about $48 \%$ of the time, while a water molecule dominates it $52 \%$ of the time. This has 


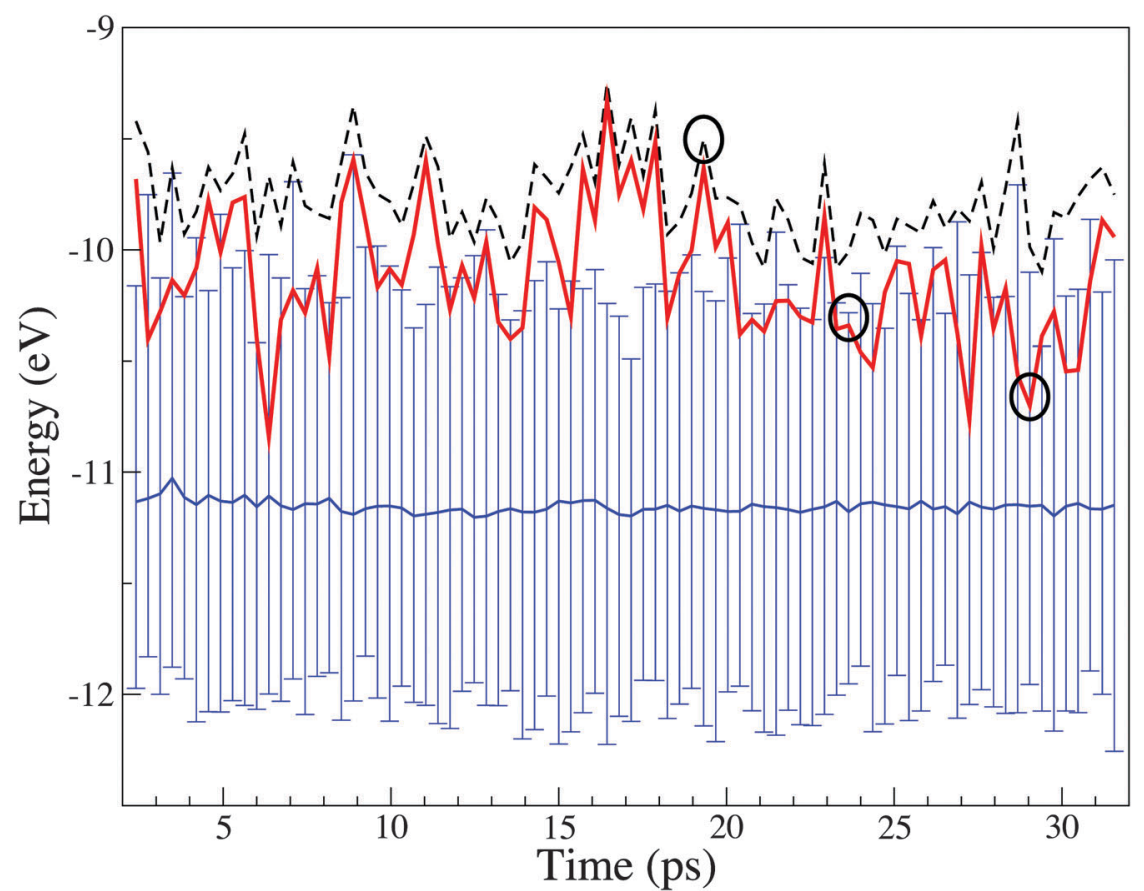

Fig. 4 Variation in energy of the chloride HOMO and highest energy occupied water band over the course of a trajectory. The dashed line refers to the system Kohn-Sham HOMO. The thick red line refers to the effective HOMO of the $\mathrm{Cl}$ anion. The continuous blue line represents the centre of the valence band of water, and the vertical lines mark its instantaneous width (conventionally indicated in Fig. 3 as $1 b_{2}$ ) representing the range of HOMO energies of the 63 water molecules in the system. The upper and lower edges of the vertical lines represent the upper and lower range of the 63 HOMO energies. The circles identified specific sampled structures.

Table 2 Average values of energy parameters for the water and chloride EMOs (in $\mathrm{eV}$ ). The numbers in brackets are for pure water ${ }^{78}$

\begin{tabular}{llll}
\hline MO & $\alpha$ & $\Delta \alpha$ & $\beta$ \\
\hline Water & & & \\
$2 \mathrm{a}_{1}$ & $-28.48(-28.30)$ & $0.45(0.43)$ & $0.78(0.58)$ \\
$1 \mathrm{~b}_{1}$ & $-15.96(-15.79)$ & $0.52(0.46)$ & $1.21(1.41)$ \\
$3 \mathrm{a}_{1}$ & $-13.11(-12.88)$ & $0.47(0.48)$ & $1.27(1.57)$ \\
$1 \mathrm{~b}_{2}$ & $-11.16(-10.90)$ & $0.44(0.42)$ & $1.01(1.11)$ \\
& & & \\
Chloride & & 0.31 & 0.71 \\
$3 \mathrm{~s}$ & -21.66 & 0.31 & 1.36 \\
$3 \mathrm{p}$ & -10.53 & 0.33 & 1.11 \\
$3 \mathrm{p}$ & -10.29 & 0.30 & 0.82 \\
$3 \mathrm{p}$ & -10.12 & & \\
\hline
\end{tabular}

implications for the instantaneous reactivity of the system. Nevertheless, this result must be treated with caution due to the propensity of BLYP to over delocalise the Kohn-Sham orbitals.

Interpreting the "energy level" or $\alpha$ data, the water $\alpha$ values in the aqueous $\mathrm{Cl}$ anion solution are similar to those of the pure water, and the same is true for $\Delta \alpha$. Moreover there is not a great spread in these values. These results indicate that the $\mathrm{Cl}$ anion, at this concentration, has only a small effect on the water orbital energy levels or thermal broadening. This is consistent with PES experiments which show no disturbance of the electronic structure (DOS) of water for iodide salts. ${ }^{98}$

From the EMO perspective the valence band of water is composed of the $1 b_{2}$ MOs of different water molecules, that is "lone pair" orbitals localised on the oxygen atoms. The experimental and the computed valence band are not substantially broadened, indicating that these orbitals remain non-bonding between water MOs. The deeper, H-bonding capable water MOs $\left(1 b_{1}\right.$ and $\left.3 a_{1}\right)$ show more broadening and therefore water-water interactions. The experimentally observed broadening of the deep $2 \mathrm{a}_{1}$ MO is not well recovered. Lone pairs of this type are generally considered to correlate electrons in dispersive interactions. Simulations including dispersion interactions have just recently become possible. ${ }^{68,99,100}$ A further application of the EMO method to a simulation in which a dispersion corrected functional has been employed, and a comparison with the results presented here would be useful for understanding the extent of dispersion effects on the electronic structure.

The thermal (environmental) broadening $\Delta \alpha$ for the $\mathrm{Cl}$ anion orbitals is $\approx 0.31 \mathrm{eV}$, smaller than that of the water bands. This may be due to fewer configurations sampled, 80 compared to the 5040 for the water orbitals, or a genuinely smaller $\Delta \alpha$ for the $\mathrm{Cl}$ anion. This indicates that the local environment for the chloride ion is more structured and that it varies less over time than for a bulk water molecule. This is consistent with the slower dynamics observed for the chloride-water compared to water-water $\mathrm{H}$-bond interactions in the bulk.

The $\mathrm{Cl}$ anion $\beta$ values are of a similar magnitude to the water molecule $\beta$ values. This means that the water-water coupling is similar to the chloride-water coupling. For both the chloride ion and water molecules the HOMO shows a reduced electronic coupling compared to the slightly lower energy EMOs. 
This indicates that interactions are not dominated by the molecular HOMO (HOMO-LUMO interactions are often drivers for chemical reactivity). In contrast to $\alpha$, the electronic broadening of the water $\beta$ values is rather sensitive to the addition of a chloride ion. $\beta$ also varies more strongly than $\Delta \alpha$ (standard deviations: $0.16,0.25,0.25$ and $0.28 \mathrm{eV}$ ).

So far the quantities analysed have been trajectory averages, however the nature of the orbital interactions between the chloride ion and solvent water molecules can be interpreted in terms of instantaneous descriptors, $\alpha, \Delta \alpha$ and $\beta$ computed for snap-shots from the trajectory, and thus we are now in a position to link a local solvation geometry to the stability of the chloride HOMO.

Several representative structures, indicated by the black circles in Fig. 4, have been selected for a more detailed analysis, Fig. 5 and Tables S2 and S3 (ESI $\dagger$ ). These snap-shots represent high, even and low energy chloride HOMOs relative to the water band edge. In Fig. 5, atoms within $12 \AA$ of the chloride ion are depicted as stick structures, hydrogen atoms within the first minimum of the $\mathrm{Cl}-\mathrm{H}$ RDF are represented as light grey spheres. Oxygen atoms within the first solvation shell boundary (defined by the first minimum in the $\mathrm{Cl}-\mathrm{O} \mathrm{RDF}$ ) are represented as spheres, two types of water molecules are identifiable; "H-bonding" water molecules that are directly H-bonding (coordinated) to the chloride ion (red spheres) and "local" water molecules (blue spheres) that are not H-bonding to the chloride ion (hydrogen atoms outside of the first minimum of the Cl-H RDF).

Fig. 5(a) shows a configuration where the chloride HOMO lies deep within the water band, i.e. when $\mathrm{Cl}$ anion is stabilized. In this case, there are six water molecules H-bonding to the $\mathrm{Cl}$ anion and no local water molecules, i.e. all of the water molecules within the first solvation shell boundary are H-bonding to the $\mathrm{Cl}$ anion. Fig. 5(b) shows a configuration in which the top of the water HOMO band and the chloride HOMO are essentially degenerate, in this case the $\mathrm{Cl}$ anion is $\mathrm{H}$-bonding to five water molecules arranged roughly in a distorted squarebased pyramid. Fig. 5(c) shows a local configuration which produces a high energy $\mathrm{Cl}$ anion $\mathrm{HOMO}$, in this case there are four water molecules $\mathrm{H}$-bonding with the $\mathrm{Cl}$ anion and two local water molecules. Thus the $\mathrm{Cl}$ anion favours coordination by six H-bonding water molecules. The lower coordination numbers are therefore likely due to the water dynamics, such that only five or even four water molecules are available.

Using the EMO method it is possible to provide a numerical value representative of the electronic effects of the chloride ion within and beyond the first solvation shell (i.e. $\beta$ ). For the individual structures selected above $\mathrm{Cl}$-water interactions have been divided into those with water within the first solvation shell ( $\beta_{1 \text { stshell-Cl }}$ ), and those with water outside the first solvation shell $\left(\beta_{\text {bulk-Cl }}\right)$. Water-water interactions have been divided into bulk water interacting with either the first solvation shell $\left(\beta_{1 \text { stshell-bulk }}\right)$, or bulk water $\left(\beta_{\text {bulk }}\right)$, Fig. 6 and Table 3. (A detailed

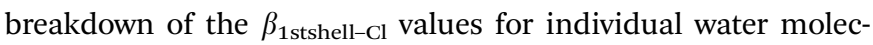
ules within the first solvation shell is given in Table S3, ESI. $\dagger$ )

The data in Table 3 show that interactions between the $\mathrm{Cl}$

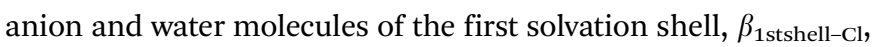
are much larger than the average water-water interactions, $\beta_{\text {bulk }} \approx 0.017 \mathrm{eV}$. However, despite a strong interaction with the chloride ion, which might be expected to reduce the interaction with bulk water molecules, these molecules maintain a large proportion of their water-water interactions, $\beta_{1 \text { stshell-bulk }} \approx$ $0.014 \mathrm{eV}$. This is a key conclusion: water molecules which are involved in $\mathrm{H}$-bonding to the chloride ion are not prevented from interacting with other water molecules.

To further investigate the decay of $\beta_{\mathrm{Cl} \text {-water }}$ away from the $\mathrm{Cl}$ anion, and thus to evaluate the extent of the electronic influence of the $\mathrm{Cl}$ anion on water molecules outside the first solvation shell, $\beta_{\text {Cl-water }}$ for water molecules within $6.0 \AA$ of the $\mathrm{Cl}$ anion have been evaluated for 10 snap-shots selected from the trajectory and plotted against the radial $\mathrm{Cl}-\mathrm{O}$ distance, Fig. 7. The decay curve has been fitted by an exponential, $\beta_{0} \mathrm{e}^{-\lambda R}$, with $\beta_{0}=13.66 \mathrm{eV}$ and $\lambda=1.40 \AA^{-1}$; and a stretched exponential, $\beta_{0} \mathrm{e}^{-\lambda_{1} R^{-\lambda_{2}}}$, with $\beta_{0}=57.85 \mathrm{eV}, \lambda_{1}=1.66 \AA^{-1}$ and $\lambda_{2}=1.08$. Despite the fact that a plane wave basis has been employed, the computed EMOs are localised on individual molecules. Hydrogenic orbitals decay exponentially with respect to the distance from the nucleus, and for simple hydrogenic orbital bond integrals, decay is exponential with respect to the internuclear separation. Thus obtaining an

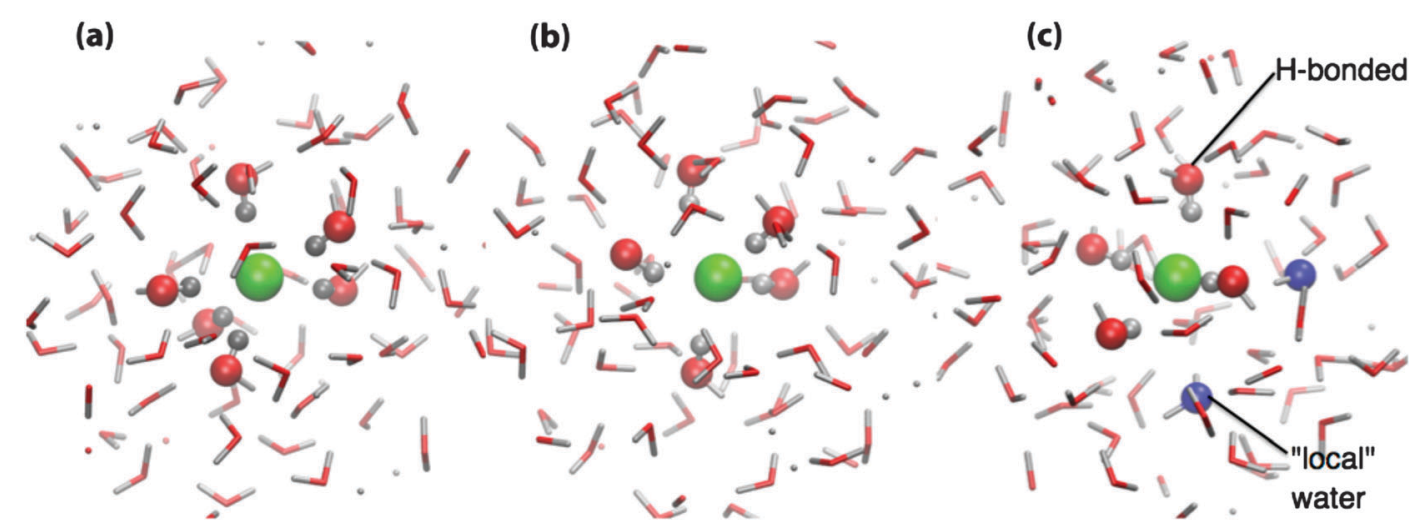

Fig. 5 Selected configurations centered on the chloride anion and showing the first solvation shell water molecules: (a) low-energy chloride HOMO, (b) intermediateenergy chloride HOMO, and (c) high-energy chloride HOMO, see the text for a description of the atoms represented as spheres. 


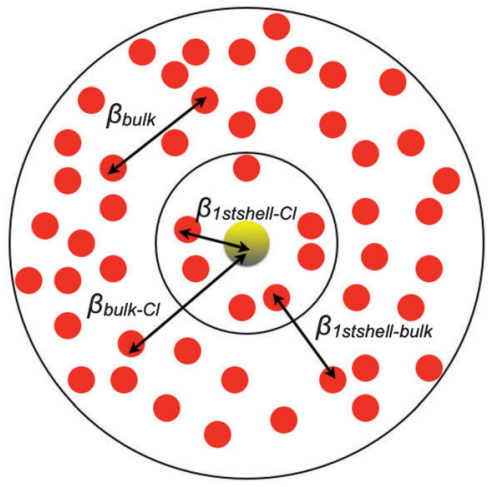

Fig. 6 Representation of electronic interaction per pair of molecules: the light (yellow) solid circle represents the $\mathrm{Cl}$ anion, the inner black circle represents the first solvation shell, and the outer black circle represents bulk water.

Table 3 EMO $\beta$ (in eV) for the structures shown in Fig. 5

\begin{tabular}{llll}
\hline & $\begin{array}{l}\text { Low Cl } \\
\text { HOMO 5(a) }\end{array}$ & $\begin{array}{l}\text { Level Cl } \\
\text { water HOMOs 5(b) }\end{array}$ & $\begin{array}{l}\mathrm{High} \mathrm{Cl}^{-} \\
\text {HOMO 5(c) }\end{array}$ \\
\hline$\beta_{1 \text { stshell-Cl }}$ & 0.179 & 0.206 & 0.137 \\
$\beta_{\text {bulk-Cl }}$ & 0.004 & 0.004 & 0.004 \\
$\beta_{1 \text { stshell-bulk }}$ & 0.014 & 0.014 & 0.013 \\
$\beta_{\text {bulk }}$ & 0.017 & 0.017 & 0.018 \\
\hline
\end{tabular}

exponential decay for beta appears to be reasonable. However, the stretched exponential is clearly more indicative of the decay of the $\beta$ integral with distance. We interpret this non-exponential decay as a manifestation of heterogeneous dynamics in our system, i.e. with individual relaxing units, for example, subsets of hydrogen bonded water molecules, or partially ordered solvation shells having individual relaxation times. ${ }^{101,102}$

Values of $\beta$ are significantly larger than 0.004 (background bulk-Cl interaction) beyond the first solvation shell. Employing the fitted equation, $\beta$ decays to the background level at $5.82 \AA$ just before the second minimum of the $\mathrm{Cl}-\mathrm{O}$ RDF (which is close to $6 \AA$ A). Thus, there is a direct electronic influence of the $\mathrm{Cl}$ anion into the second solvation shell, but this effect does not extend beyond the second solvation shell. The similarity of the water-water $\beta_{1 \text { stshell-bulk }}$ and $\beta_{\text {bulk }}$ quantities also indicate that indirect electronic effects beyond the second solvation shell are minimal. While these results show that the effect of the $\mathrm{Cl}$ anion does not extend beyond the second solvation shell, these data should be treated as a maximum estimate for the water-Cl anion electronic coupling due to the BLYP functional exaggerating electronic delocalisaton.

The $\mathrm{Cl}$ anion can "mimic" a water molecule effectively in terms of matching the electronic overlap with other water molecules. In Fig. 7 the largest $\beta$ values are for interaction of first solvation shell water molecules with the $\mathrm{Cl}$ anion (0.25), and analysis of the direct water-water interactions in bulk water also gives strong beta values (0.26). It might be considered that a large orbital coupling should result in some charge transfer. This would be the case for normal bonding interactions where one fragment orbital is occupied and the other unoccupied. However, in this case both fragment orbitals are occupied and significant redistribution of electron

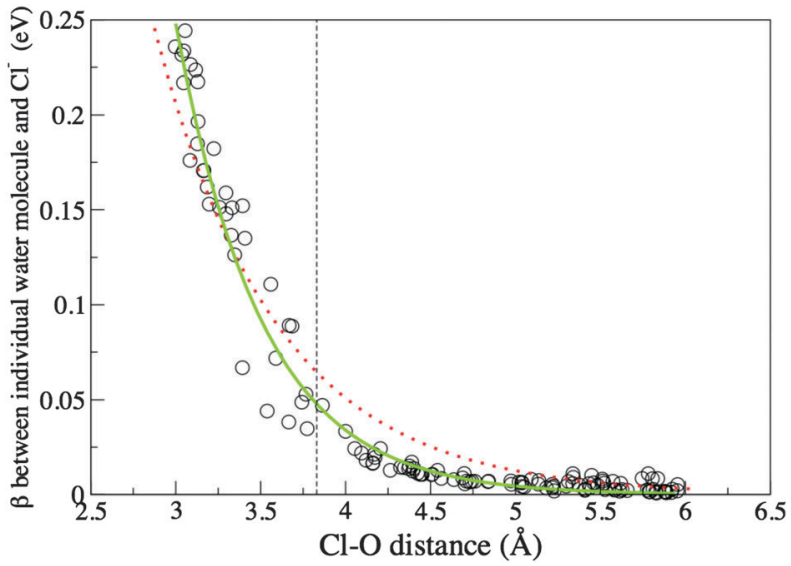

Fig. $7 \quad \beta$ as a function of $\mathrm{Cl}-\mathrm{O}$ distance. Circles are calculated data points, the dotted (red) line is the fitted exponential, the continuous (green) line is the fitted stretched exponential, and the dashed line identifies the first minimum of the Cl-O RDF.

density from one molecular fragment to another is not expected.

\subsection{Local solvation around the $\mathrm{Cl}$ anion}

The coordination number and the local arrangement of the water molecules around the $\mathrm{Cl}$ anion is of particular interest. Based on our simulations the $\mathrm{Cl}$ anion is on average surrounded by $5-6$ water molecules, however, the $\mathrm{Cl}-\mathrm{O}$ coordination number (6.3) is essentially one more than the $\mathrm{Cl}-\mathrm{H}$ derived coordination number (5.1). The larger coordination number for oxygen compared to hydrogen has also been found experimentally at the $\mathrm{Cl}$ :water 1:83 ratio, this was ascribed to the presence of an interstitial water molecule. ${ }^{20}$

Inspection of the trajectory shows that the hydrogen atom coordination number reflects the number of water molecules $\mathrm{H}$-bonding to the $\mathrm{Cl}$ anion. The extra oxygen atom or atoms belong to "local" water molecules. Local water molecules have oxygen atoms that lie within the first minimum of the $\mathrm{Cl}-\mathrm{O}$ RDF but hydrogen atoms that are not within the first $\mathrm{Cl}-\mathrm{H}$ RDF minimum.

Typically there are 1-4 extra local water molecules, and they take on a variety of conformations. In Fig. 8 water molecules within $8.0 \AA$ of the $\mathrm{Cl}$ anion are depicted, those lying within the $\mathrm{Cl}-\mathrm{O}$ boundary are represented as ball and stick structures, blue oxygen atoms labelled $\mathrm{L}$ belong to local water molecules, purple water molecules labelled B are not within the first solvation shell but have been identified to highlight specific water molecules that are H-bonding with the local water molecules, the remaining ball and stick water molecules (with red oxygen atoms) are those that $\mathrm{H}$-bond to the $\mathrm{Cl}$ anion, $\mathrm{H}$-atoms within the $\mathrm{Cl}-\mathrm{H}$ boundary are represented as dark grey.

For example, a local water molecule can $\mathrm{H}$-bond to the surrounding bulk water, rotated such that the $\mathrm{H}$-atoms are facing away from the chloride ion and interacting with bulk water, Fig. 8(a). Fig. 8(b) shows multiple local water molecules $\mathrm{H}$-bonding to each other, and Fig. 8(c) shows local water molecules $\mathrm{H}$-bonding to water molecules $\mathrm{H}$-bonding with the 


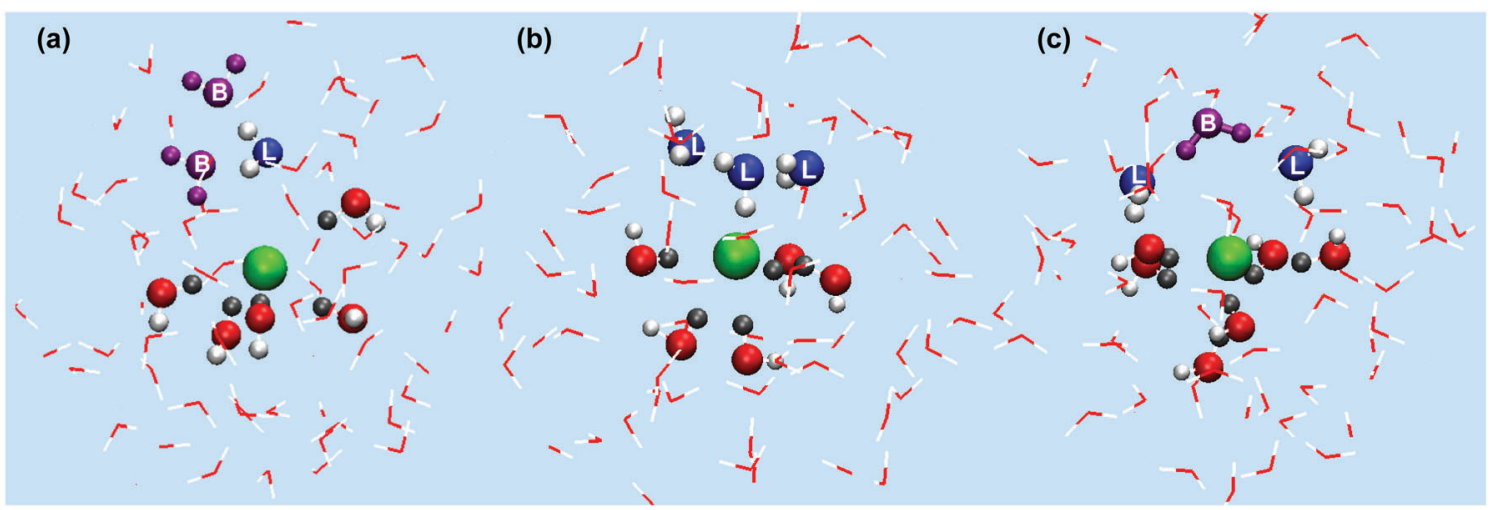

Fig. 8 Example structures showing the local arrangement of water molecules in the first solvation shell around the $\mathrm{Cl}$ anion, see the main text for a full description. (a) Single local water $\mathrm{H}$-bonding with bulk water, (b) multiple local water molecules mutually $\mathrm{H}$-bonding, (c) two local water molecules $\mathrm{H}$-bonding to coordinated water molecules.

$\mathrm{Cl}$ anion. Visual inspection of the trajectory shows that first solvation shell water molecules predominantly collect on one side of the $\mathrm{Cl}$ anion, often but not always, the local water molecules collect on the opposite side, H-bonding to each other or bulk water. The $\mathrm{Cl}$ anion $\mathrm{H}$-bonding water molecules tend not to H-bond with each other, but form H-bonds with other bulk water molecules.

The number of water molecules associated with the chloride ion has been evaluated over time. Fig. 9 shows a histogram of the coordination numbers collected over the trajectory based on the average $\mathrm{Cl}-\mathrm{O}$ and $\mathrm{Cl}-\mathrm{H}$ distances defined in Table 1 . The $\mathrm{H}$-atom distribution is narrower and dominated by a coordination number of five. From the $\mathrm{Cl}-\mathrm{O}$ distribution it is evident that the $\mathrm{Cl}$ anion is associated $\approx 25 \%$ of the time with 5 water molecules and $\approx 40 \%$ of the time with 6 water molecules; this means that there is regularly one local water molecule present in the first solvation shell. However, larger numbers of local water molecules can occur; $\approx 30 \%$ of the time there are $7-9$ water molecules within the first solvation shell.

The distribution of $\mathrm{O}-\mathrm{Cl}-\mathrm{O}$ angles in the first solvation shell is shown in Fig. 10. Fig. 10 shows a maximum around $80^{\circ}$, and small peak around $46^{\circ}$, consistent with water molecules in the first solvation shell collecting on one side of the chloride ion. A similar asymmetric distribution has been found for $a b$ initio MD simulations of aqueous bromide or chloride ions, ${ }^{67,103}$ and neutron diffraction studies have found a peak at about $70^{\circ} .{ }^{20} \mathrm{It}$ is also well known that in small clusters (or at an interface) the $\mathrm{Cl}$ ion prefers an unsymmetrical solvation shell. ${ }^{65}$

To investigate differences with respect to gas phase clusters, a small sample of representative calculations was carried out at the B3LYP/cc-pVTZ and MP2/cc-pVTZ levels for selected conformers of $\mathrm{Cl}\left(\mathrm{H}_{2} \mathrm{O}\right)_{5}{ }^{-}$and $\mathrm{Cl}\left(\mathrm{H}_{2} \mathrm{O}\right)_{6}{ }^{-}$(Tables $\mathrm{S} 5$ and $\mathrm{S} 6$ and Fig. S10-S12, ESI $\dagger) \cdot\left[\mathrm{Cl}\left(\mathrm{H}_{2} \mathrm{O}\right)_{5}\right]^{-}$is slightly more stable than $\left[\mathrm{Cl}\left(\mathrm{H}_{2} \mathrm{O}\right)_{4} \cdot \mathrm{H}_{2} \mathrm{O}\right]^{-}$by $\approx 5 \mathrm{~kJ} \mathrm{~mol}^{-1}$. $\left[\mathrm{Cl}\left(\mathrm{H}_{2} \mathrm{O}\right)_{6}\right]^{-}$with all the water molecules H-bonding in a ring below the chloride ion was unstable (Fig. S12a, ESI $\dagger$ ) and a distorted octahedral geometry was preferred (Fig. S11a, ESI $\dagger$ ). This is consistent with the lowest energy $\mathrm{Cl}$ anion solvation shell structure extracted from the trajectory. Multiple structures with $\left[\mathrm{Cl}\left(\mathrm{H}_{2} \mathrm{O}\right)_{4} \cdot\left(\mathrm{H}_{2} \mathrm{O}\right)_{2}\right]^{-}$and

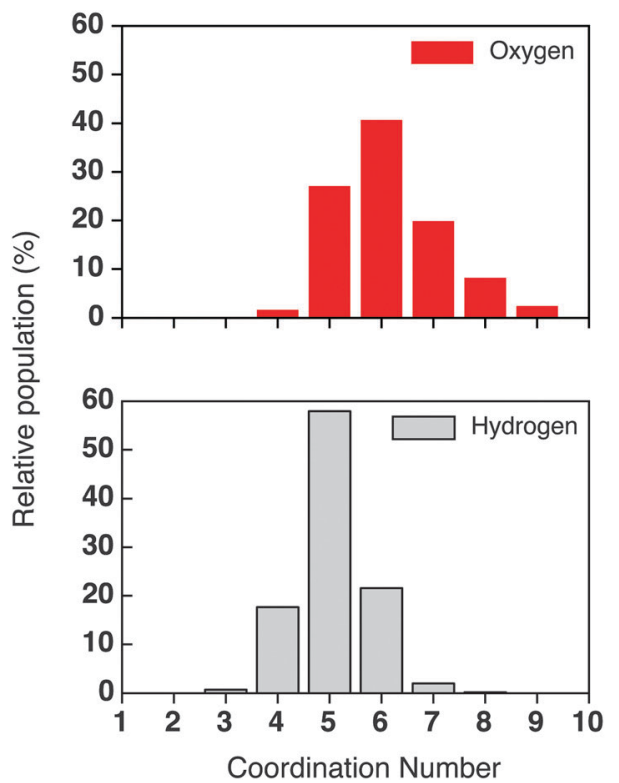

Fig. 9 Histogram of the coordination number based on the $\mathrm{Cl}-\mathrm{O}$ and $\mathrm{Cl}-\mathrm{H}$ distances for $\mathrm{Cl}^{-}$in water solution.

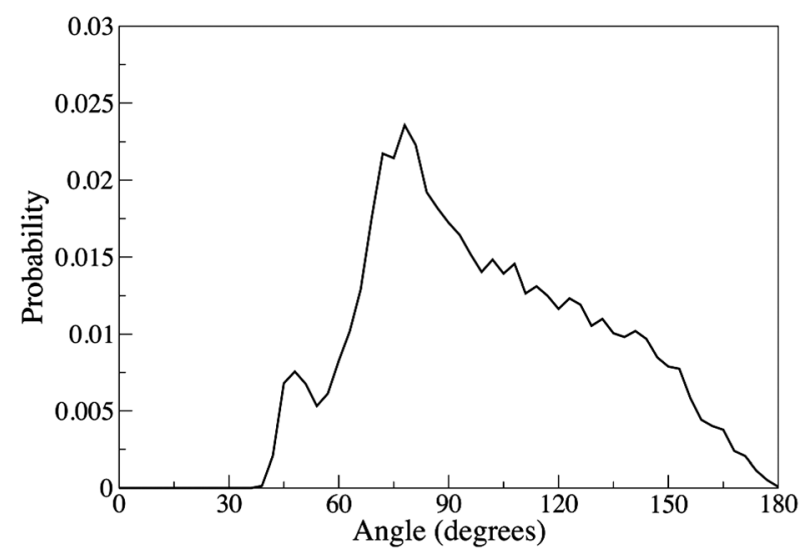

Fig. 10 Distribution of the $\mathrm{O}-\mathrm{Cl}-\mathrm{O}$ angle calculated within the first minimum of the $\mathrm{Cl}-\mathrm{O}$ RDF. 
$\left[\mathrm{Cl}\left(\mathrm{H}_{2} \mathrm{O}\right)_{3} \cdot\left(\mathrm{H}_{2} \mathrm{O}\right)_{3}\right]^{-}$arrangements were found, some close in energy to the most stable structure (between 0 and $20 \mathrm{~kJ} \mathrm{~mol}^{-1}$ ). Thus, structures with bridging and second solvation shell water molecules are also evident in gas-phase clusters. The stability of an additional $\mathrm{Cl}$-water $\mathrm{H}$-bond is similar to that obtained from a water-water $\mathrm{H}$-bond. A key difference is that the first solvation shell water molecules in the gas phase clusters are forced to form $\mathrm{H}$-bonds with each other, while the liquid phase first solvation shell water molecules prefer to H-bond with the second solvation shell or bulk water molecules. More importantly "local" water molecules not H-bonding to the $\mathrm{Cl}$ anion cannot occur for the clusters, as they need to be stabilised by $\mathrm{H}$-bonding to second solvation shell water molecules.

The HOMO, LUMO energy levels and HOMO-LUMO gap are presented in Tables S7 and S8 (ESI $\dagger$ ). The energy levels from the MP2 calculations are closer to those obtained experimentally for the liquid, however B3LYP provides a better estimate of the HOMO-LUMO gap. The position of the $\mathrm{Cl}$ anion HOMO relative to the water valence band edge (VBE) in the B3LYP and MP2 calculations for these selected conformers has also been computed (Tables S7 and S8, ESI $\dagger$ ). In all cases, the HOMO of the clusters is of 3-fold degeneracy on the $\mathrm{Cl}$ anion, and the VBE of water lies below the $\mathrm{Cl}$ anion HOMO. For example, for the most stable conformer of $\mathrm{Cl}\left(\mathrm{H}_{2} \mathrm{O}\right)_{6}{ }^{-}, \mathrm{MP} 2$ calculations predict that the HOMO $(-7.04 \mathrm{eV})$ of the $\mathrm{Cl}$ anion is $2.71 \mathrm{eV}$ above the $\mathrm{VBE}$ of water $(-9.75 \mathrm{eV})$; B3LYP calculations predict the HOMO of the $\mathrm{Cl}$ anion and the water $\mathrm{VBE}$ to be $-3.40 \mathrm{eV}$ and $-4.75 \mathrm{eV}$, respectively; producing an energy gap of $1.35 \mathrm{eV}$. Thus, the B3LYP prediction is compared more favourably to the corresponding experimental energy gap $(1.1 \mathrm{eV})^{97}$ within the liquid.

\subsection{Dipole moments}

The $\mathrm{Cl}$ anion is polarized within the bulk liquid; directional $\mathrm{H}$-bonds are formed, and water molecules "group" on one side of the $\mathrm{Cl}$ anion. These interactions are not static, $\mathrm{H}$-bonds are breaking and reforming in new patterns, and the polarisation of the $\mathrm{Cl}$ anion shifts. Not only does the water respond to the anion, but the anion responds to the water. Thus, a robust description of the dipole and electronic polarizability is important for studying ionic systems, and this is naturally taken into account by the electronic wavefunction of the $a b$ initio MD.

Dipole moments have been computed employing a Wannier function analysis. The Wannier orbitals are obtained from the Kohn-Sham orbitals via a unitary transformation, where the spread of the orbitals is minimized iteratively. ${ }^{104}$ Using the centres of the Wannier functions and associated charges, the dipole moment of a water molecule can be calculated using the classical dipole moment formula. This type of analysis has previously been employed to analyse dipole moments. ${ }^{70,103,105-110}$

The calculated dipole moments of the water molecules inside and outside the first solvation shell are $3.05 \pm 0.14$ and $3.10 \pm 0.06 \mathrm{D}$, respectively, and thus they are essentially equal within the error limits. Our results are consistent with those from previous CPMD studies ${ }^{67,68,81,103,111}$ and an experimentally based estimate for the dipole moment of pure water, $2.9 \pm 0.6 \mathrm{D}^{112}$ The halide and concentration of the other simulations does vary, however even in concentrated solutions the average dipole moment of water does not appear to vary by a large amount, for example an average dipole moment of $3.07 \mathrm{D}$ has been obtained for water in a $14 \mathrm{~mol} \mathrm{~L}^{-1}$ solution of LiCl. ${ }^{113}$ The calculated dipole moment of the $\mathrm{Cl}$ ion is $0.79 \pm$ $0.39 \mathrm{D}$. This value compares favourably with the results from other CPMD simulations $0.82 \pm 0.32 \mathrm{D},{ }^{68}$ and the value from a combined quantum chemical and statistical model of $0.35 \pm$ $0.14 \mathrm{D}$ seems to be low. ${ }^{114}$

Heuft and Meijer found significant fluctuations in the chloride dipole moment, however only 13 configurations were sampled, in most of these the dipole was found to be $\approx 1 \mathrm{D}$ but there were two configurations with much larger dipole moments, up to 4 and 5 D. ${ }^{67}$ We have sampled 80 configurations, and the chloride ion dipole moment deviates significantly from zero over the simulation, Fig. 11. However, we found no dipole moments of magnitude greater than $1.75 \mathrm{D}$. A similar study for the bromide ion found a dipole moment of 0.95 with a standard deviation of $0.8 \mathrm{D}$, and a maximum dipole of $\approx 1.75 \mathrm{D}^{103}$

Importantly, the average magnitude of the chloride ion dipole is $\approx 3$ times less than that of the water molecule it "replaces". The chloride ion is less "polarized" than a neutral water molecule. This is consistent with water molecules in small chloride ion water clusters having a smaller dipole moment; the chloride ion is less polarizing than another water molecule. For example, the dipole moment of water in small clusters $\mathrm{Cl}\left(\mathrm{H}_{2} \mathrm{O}\right)_{n}(n=1-5)$ has been found to be in the region of 2.5-2.6 D. ${ }^{70}$ This difference has been taken to mean that dipole moments for water molecules in the first solvation shell are dominated by water-water rather than ion-water interactions.

In the past, the ability of the chloride ion to form "a large dipole" has been suggested as the driving force for the formation of stable cluster geometries with the water molecules collected on one side of the ion. ${ }^{63}$ However, the small dipole moment determined for the chloride ion relative to the water molecules does not align well with this rationalisation. The polarizability of the chloride ion relative to water molecules must play a significant role. The small dipole moment induced

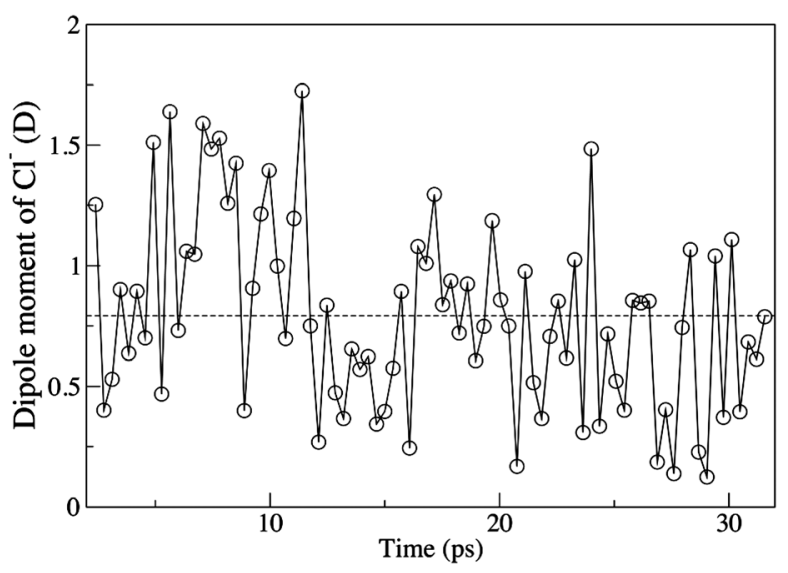

Fig. 11 Time evolution of the dipole moment of the aqueous chloride ion, the dashed line is the average dipole moment. 
on the chloride ion may not be the only driver for an asymmetric solvation shell. A highly symmetric, non-polarised cluster, or first solvation shell is not favoured entropically, and a larger range of configurations and H-bonding motifs can occur if the chloride ion takes a polarised configuration. The stabilisation of water-water $\mathrm{H}$-bonds is important, and the small number of water molecules in a cluster means that they must collect on one side of the chloride in order to form water-water $\mathrm{H}$-bonds. This limitation is removed in the aqueous system where H-bonding with bulk (second solvation shell) water molecules is possible, and as shown above favoured.

As the dipole moment of the whole system is zero, fluctuations of the chloride ion dipole must be accommodated by the water environment. Because the dipole moments of first solvation shell water molecules do not differ significantly from those in the bulk water, these adjustments must be small. The results presented here show that the average water dipole moment is not strongly affected by the presence of the chloride ion. Fig. S13 (ESI $\dagger$ ) shows how a water molecule can maintain a directional $\mathrm{H}$-bond to the chloride ion while still allowing the water molecule to re-orientate around the $\mathrm{H}$-bonded $\mathrm{H}-\mathrm{O}$ bond vector, leaving the molecular dipole moment free to re-orientate. Such effects result in different dynamics for $\mathrm{H}$-bonds and dipole moments. ${ }^{115}$

\subsection{Exchange events}

A residence time reflects the time a water molecule remains $\mathrm{H}$-bonded to the chloride ion. Residence times computed for water near a chloride ion can vary depending on the method employed, for example a time constant of $4.5 \mathrm{ps}$ has been obtained from classical MD (employing a non-polarizable potential), ${ }^{56}$ while including polarization effects increases this to 2-3 times longer, ${ }^{24}$ and employing ab initio MD a residence time of $12 \pm 3$ ps has been obtained. ${ }^{67}$ The spectral diffusion time for the $\mathrm{OH}$ stretch (i.e. fluctuations in the length of the $\mathrm{O}-\mathrm{H}$ bond) are thought to be related to the residence time of the water molecule. Using femtosecond pump-probe IR spectroscopy the time constant for bulk water is $0.5 \pm 0.2 \mathrm{ps}$ and for water in the first solvation shell of a chloride anion was determined as $12 \pm 3$ ps. $^{52}$ Using self-diffusion constants determined from NMR the residence time for a water molecule in the hydration shell of chloride is $15 \mathrm{ps}^{24}$ This match between computed and experimental time constants indicates that the $a b$ initio MD is recovering the dynamics of water molecules around a chloride anion.

Events such as water molecules leaving or entering the first solvation shell are rare, but can be monitored by examining the Cl-O radial distances of the water molecules (Fig. 12 shows a single event, Fig. S14 (ESI $\dagger$ ) shows the $\mathrm{Cl}-\mathrm{O}$ radial distance for all the water molecules along the course of the trajectory). Two time scales are evident: rapid oscillations which reflect $\mathrm{H}$-bonds breaking and reforming, and slower dynamics which show when molecules cross the first or second solvation shell boundaries. During our simulation 10 water molecules moved between the first and the second solvation shells, and both timescales are readily observable in the animated trajectory.

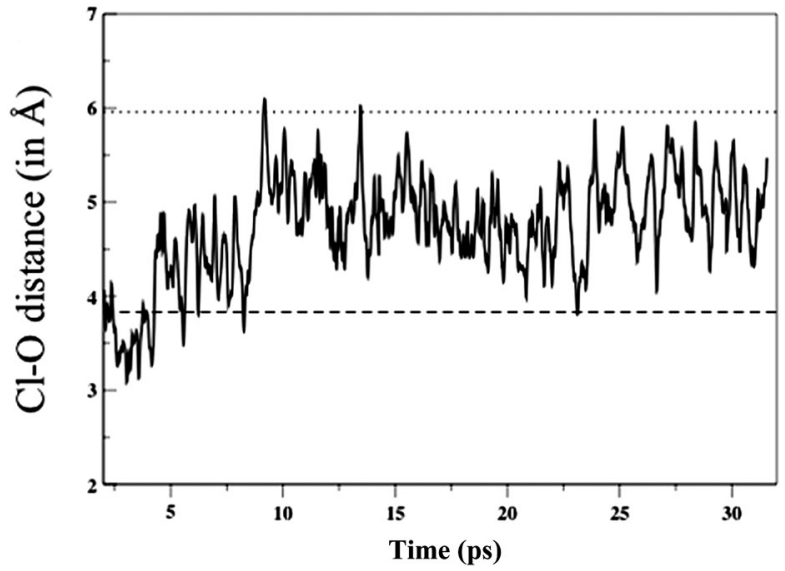

Fig. 12 Time evolution of the $\mathrm{Cl}-\mathrm{O}$ distance for a single water molecule, the dashed lines are the first and second solvation shell boundaries.

There are experimental decay constants supporting these observations; water molecules break and reform H-bonds (2.6 ps), and reorientate $(9.6 \mathrm{ps})$ much faster than they dissociate from the anion $(\approx 12 \mathrm{ps}) .{ }^{51,52}$

The movements of the ten water molecules exchanging around the chloride ion can be divided into three categories, representative examples of the evolution of the radial distances are shown in Fig. S15 (ESI $\dagger$ ). (i) A water molecule stays in the first solvation shell for $\approx 6 \mathrm{ps}$, then leaves, remains in the second solvation shell or comes back to the first solvation shell, there are three molecules in this category. (ii) A water molecule enters the first solvation shell briefly and then leaves, there are five molecules in this category. (iii) A water molecule enters the first solvation shell and remains in the shell for the rest of the simulation, there are two molecules in this category. In some of these cases a water molecule has entered the first solvation shell and a short time later a different water molecule leaves. Overall one water molecule remained in the first solvation shell over the entire trajectory, four water molecules moved into the first solvation shell and stayed, three water molecules left and returned, and two water molecules moved out of the first solvation shell not to return.

Inspection of the trajectory shows that water molecules with a $\mathrm{H}$-bond to the $\mathrm{Cl}$ anion tend to remain close to the $\mathrm{Cl}$ anion. The local water molecules (those within the first Cl-O boundary but not $\mathrm{H}$-bonding to the $\mathrm{Cl}$ anion) are more dynamic. In analogy with the phenomenon of "slaving" observed in protein dynamics, ${ }^{101,102}$ these water molecules may respond more readily to bulk water fluctuations. Hence, an analysis based purely on the $\mathrm{Cl}-\mathrm{O}$ radial distance of water molecules within the first solvation shell may not provide sufficient resolution, there may be two time-scales for water molecules around the $\mathrm{Cl}$ anion, one for directly H-bonding and one for local water molecules. In this case a dual selection criteria including $\mathrm{Cl}-\mathrm{H}$ and $\mathrm{Cl}-\mathrm{O}$ radial cut-off may offer more insight. The EMO $\beta$ analysis indicates that while the local water molecules $(\beta \approx 0.05)$ are not as strongly interacting as the $\mathrm{H}$-bound water $(\beta \approx 0.20)$ they are interacting with the chloride ion more than bulk water molecules $(\beta \approx 0.004)$. 
Inspection of the motions occurring around the $\mathrm{Cl}$ anion (Fig. S16, ESI $\dagger$ ) shows small changes in distances and angles. Motion of the H-bound water molecules is more localised, and a "circling" pattern is evident. Motion of the water molecules between the $\mathrm{Cl}-\mathrm{O}$ boundary and $5.0 \AA$ from the $\mathrm{Cl}$ anion is less constrained and "linear" translation features are more pronounced. Motion of the local water molecules lies between these two extremes: circling or linear translation. Local water molecules tend to cross and recross the $\mathrm{Cl}-\mathrm{O}$ boundary relatively freely, consistent with the breaking and reforming of water-water $\mathrm{H}$-bonds. However, they also tend to stay within $5.0 \AA$ A of the $\mathrm{Cl}$ anion.

The formation and breaking of $\mathrm{H}$-bonds and reorientation (angular changes) of water molecules are known to be related in a complex way. Unraveling the information obtained from simulation and experimental studies therefore becomes difficult. The mechanism for breaking and reforming of H-bonds in aqueous solution has not been established. In a "diffusive" mechanism, the $\mathrm{O} \cdots \mathrm{H}-\mathrm{O} \mathrm{H}$-bond can break and then the dangling $\mathrm{H}-\mathrm{O}$ subsequently undertakes small uncorrelated/ random angular steps, finding some time later another partner to H-bond with. In the "angle hopping"' mechanism, two water molecules become equidistant from the solute water molecule, one $\mathrm{H}$-bond breaks and almost instantaneously another $\mathrm{H}$-bond forms via a large angle jump, the mechanism is concerted. ${ }^{115,116}$ In addition, a large angle rotation can take a water molecule from the first solvation shell into the second solvation shell changing the coordination number of the anion. ${ }^{117}$ The motions we observe in the water molecules around the chloride ion are not easily separable into translational $v s$. angular components and cannot readily be used to obtain information on either of these models for H-bonding.

Thus these findings support a picture of highly mobile water molecules in the $\mathrm{Cl}$ anion first solvation shell. Water molecules are either relatively strongly bound to the anion by hydrogen bonds, or are only weakly kept in place by dipole interactions or by hydrogen bonds with other water molecules. The instantaneous dynamics of the local water molecules can therefore be strongly coupled with the fluctuation of molecules belonging to the second and subsequent solvation shells.

\section{Conclusions}

We have performed ab initio MD simulations and a geometric and orbital analysis of a $\mathrm{Cl}$ anion solution equivalent to a concentration of $0.88 \mathrm{~mol} \mathrm{~L}^{-1}$. The EMO analysis has allowed us to gain valuable insight into the electronic structure of a solvated ion immersed in solute orbitals. Using the BLYP functional we observe an oscillating evolution of the chloride ion HOMO at the band edge of the highest occupied water band. The robustness of this result is hard to evaluate because of the self-interaction error of BLYP, which may over delocalise the chloride states and position their energies incorrectly relative to the water VBM. However, as also evident from the experimental PES the $\mathrm{Cl}$ anion HOMO band is broadened and overlaps the water band edge. The difference in energy is of the order of $1 \mathrm{eV}$, which is also the order of the anticipated error from employing the BLYP functional. These results provide a good indication for the maximum extent of electronic mixing, the EMO method is general and can, in the future, be applied to functionals that have the self-interaction and long range dispersion corrected.

Comparison with pure water showed that the $\mathrm{Cl}$ anion has little effect on the average water electronic structure. Moreover, the $\mathrm{Cl}$ anion has a similar orbital energy distribution and electronic coupling compared to the water molecules. In terms of electronic structure the $\mathrm{Cl}$ anion is "water-like". However, the average magnitude of the $\mathrm{Cl}$ anion dipole is approximately three times less than that of the water molecule it "replaces". The EMO and cluster analysis showed that the $\mathrm{Cl}$ anion prefers a symmetric six coordinate environment with a low dipole moment. However, the coordinating water molecules would probably prefer the $\mathrm{Cl}$ anion to mimic a water molecule better, and have a higher dipole moment. We found that the potential for multiple structural variations (increased entropy) and the facilitation of water-water $\mathrm{H}$-bonds also favour asymmetric solvation. While water molecule $\mathrm{H}$-bond dynamics are reduced when H-bonding to a $\mathrm{Cl}$ anion, there appears to be little restriction on the dipole moment of water molecules within the first solvation shell, and the computed dipole moments for water molecules inside or outside of the first solvation shell are the same.

While the ionic nature of the $\mathrm{Cl}$ anion does not induce a strong response in the solvating water dipoles, the $\mathrm{Cl}$ anion does have an (electronic) effect beyond the first solvation shell via $\mathrm{Cl}-$ water electronic coupling. $\beta_{1 \text { stshell-Cl }}$ interactions are much larger than the average $\beta_{\mathrm{Cl}-\text { bulk }}$ or water-water interactions. Nevertheless, $\beta$ values decay to background levels within the second solvation shell. This result adds further information to the debate on the long range effects of fully solvated ions. $\mathrm{H}$-bonding with the $\mathrm{Cl}$ anion does not interfere with the waterwater H-bonding, and $\beta_{1 \text { stshell-bulk }}$ and $\beta_{\text {bulk }}$ are similar.

The local solvation structure was examined in more detail using traditional descriptors. Our initial investigation was driven by an observed difference in the $\mathrm{Cl}-\mathrm{O}$ and $\mathrm{Cl}-\mathrm{H}$ coordination numbers (6.3 and 5.1 respectively). This led to a differentiation between two types of water molecule within the first solvation shell. There are on average 5 water molecules H-bonding with the $\mathrm{Cl}$ anion, and in addition, 1-4 local water molecules which have oxygen atoms within the first minimum of the $\mathrm{Cl}-\mathrm{O}$ RDF but hydrogen atoms that are not within the first minimum of the $\mathrm{Cl}-\mathrm{H}$ RDF. The H-bonding water molecules tend to be distributed on one side of the $\mathrm{Cl}$ anion, however they do not $\mathrm{H}$-bond with each other but with water molecules in the second solvation shell. The local water molecules exhibit a variety of structural motifs, in addition their position is not as localised as that of the coordinating water molecules. They can bridge coordinated water molecules, H-bond with second solvation shell water molecules or they can form a mutually H-bonded group. There is a reasonable chance that the local water molecules will be on the opposite side of the $\mathrm{Cl}$ anion from the coordinated water molecules. 
The $\mathrm{Cl}$ anion appears to be more stable when symmetrically solvated by $6 \mathrm{H}$-bonding water molecules (both as a cluster and in solution), and the solution HOMO is driven up as this number decreases. The EMO analysis, similarity of the water and $\mathrm{Cl}$ anion electronic structure, cluster calculations, and spread of coordination numbers around 4-7 indicate that there is a fine balance between $\mathrm{Cl}$-water and water-water $\mathrm{H}$-bonding and orbital interactions. Thus, we can rationalise the flexibility of the chloride solvation shell structure (particularly the wide range of coordination numbers obtained experimentally) and the placement of the chloride ion near the centre of the Hofmeister series.

We also observed, in contrast to the clusters, that water molecules in the first solvation shell tend not to H-bond with each other but to bulk water molecules. These results indicate that the $\mathrm{Cl}$ anion solvation structure is highly influenced by the dynamics of the surrounding water, which appears to favour five asymmetrically distributed $\mathrm{H}$-bonding water molecules within the first solvation shell (the $\mathrm{Cl}-\mathrm{H}$ coordination number is five $\approx 60 \%$ of the time) and a number of local water molecules. Within the first solvation shell the solvating and local water molecules have different dynamics. Visual inspection of the trajectory and monitoring of the evolution of the radial $\mathrm{Cl}-\mathrm{O}$ distance for all water molecules showed that the $\mathrm{H}$-bonding water associated with the chloride ion for longer periods, while the local water molecules are more mobile. $\mathrm{Cl}$ anion H-bonding water molecules have a slower, more circular motion, while local water molecules had a propensity for a more linear motion. Ten molecules left or entered the first solvation shell, however rapid oscillations and the complexity of the coupled motions inhibited the identification of a clear "reaction" coordinate.

We have demonstrated the potential of the EMO method as an interpretative tool to investigate solvation at a fundamental, quantum mechanical level. We have characterised the structural and electronic factors determining the effect of a chloride ion on the presence of the surrounding polarizable water solvent, and vice versa the effect of the water on the chloride ion.

\section{Acknowledgements}

L.G. and P.H. acknowledge support from the Leverhulme Trust (F/07 058/BB). P.H. acknowledges The Royal Society for a RSURF. We sincerely thank Prof. Michiel Sprik for his contributions and stimulating discussions.

\section{References}

1 E. M. Knipping, M. J. Lakin, K. L. Foster, P. Jungwirth, D. J. Tobias, R. B. Gerber, D. Dabdub and B. J. FinlaysonPitts, Science, 2000, 288, 301.

2 C. W. Spicer, E. G. Chapman, B. J. Finlayson-Pitts, R. A. Plastridge, J. M. Hubbe, J. D. Fast and C. M. Berkowitz, Nature, 1998, 394, 353.

3 K. L. Foster, R. A. Plastridge, J. W. Bottenheim, P. B. Shepson, B. J. Finlayson-Pitts and C. W. Spicer, Science, 2001, 291, 471.
4 B. J. Finlayson-Pitts, Phys. Chem. Chem. Phys., 2009, 11, 7760 .

5 J. Walker, J. Wills and J. Reid, Weather, 2011, 66, 165.

6 S. Solomon, M. M. D. Qin, Z. Chen, M. Marquis, K. B. Averyt, M. Tignor and H. L. Miller, Climate Change 2007: The Physical Science Basis, Contribution of Working Group I to the Fourth Assessment Report of the Intergovernmental Panel on Climate Change (IPCC), Cambridge University Press, Cambridge, UK and New York, NY, 2007.

7 NASA, http://science.nasa.gov/earth-science/oceanography/ physical-ocean/salinity/, 2011.

8 H. E. Beekman, H. G. M. Eggenkamp and C. A. J. Appelo, Appl. Geochem., 2011, 26, 257.

9 D. F. Parsons, M. Bostrom, P. L. Nostro and B. W. Ninham, Phys. Chem. Chem. Phys., 2011, 13, 12352-12367.

10 K. J. Tielrooij, S. T. van der Post, J. Hunger, M. Bonn and H. J. Bakker, J. Phys. Chem. B, 2011, 115, 12638-12647.

11 F. Hofmeister, Naunyn-Schmiedeberg's Arch. Pharmacol., 1888, 24, 247-260.

12 Y. Zhang, S. Furyk, D. E. Bergbreiter and P. S. Cremer, J. Am. Chem. Soc., 2005, 127, 14505.

13 Y. Zhang and P. S. Cremer, Curr. Opin. Chem. Biol., 2006, 10, 658-663.

14 Y. Marcus, Pure Appl. Chem., 2010, 82, 1889-1899.

15 Y.-S. Lin, B. M. Auer and J. L. Skinner, J. Chem. Phys., 2009, 131, 144511.

16 D. J. Tobias and J. C. Hemminger, Science, 2008, 319, 1197-1198.

17 P. Jungwirth and D. Tobias, Chem. Rev., 2006, 106, 1259-1281.

18 M. D. Baer, I.-F. W. Kuo, H. Bluhm and S. Ghosal, J. Phys. Chem. B, 2009, 113, 15843-15850.

19 D. Paschek and R. Ludwig, Angew. Chem., Int. Ed., 2011, 50, 352-353.

20 R. Mancinelli, A. Botti, F. Bruni, M. A. Ricci and A. K. Soper, J. Phys. Chem. B, 2007, 111, 13570-13577.

21 R. Mancinelli, A. Botti, F. Bruni, M. A. Ricci and A. K. Soper, Phys. Chem. Chem. Phys., 2007, 9, 2959-2967.

22 A. Botti, F. Bruni, S. Imberti, M. Ricci and A. Soper, J. Chem. Phys., 2004, 120, 10154-10162.

23 A. Botti, F. Bruni, S. Imberti, M. Ricci and A. Soper, J. Chem. Phys., 2004, 121, 7840-7848.

24 H. J. Bakker, Chem. Rev., 2008, 108, 1456-1473.

25 A. Omta, M. Kropman, S. Woutersen and H. Bakker, J. Chem. Phys., 2003, 119, 12457-12461.

26 A. Omta, M. Kropman, S. Woutersen and H. Bakker, Science, 2003, 301, 347-349.

27 K. J. Tielrooij, N. Garcia-Araez, M. Bonn and H. J. Bakker, Science, 2010, 328, 1006-1009.

28 K. D. Collins, G. W. Neilson and J. E. Enderby, Biophys. Chem., 2007, 128, 95-104.

29 C. Cappa, J. Smith, B. Messer, R. Cohen and R. Saykally, J. Phys. Chem. B, 2006, 110, 5301-5309.

30 J. D. Smith, R. J. Saykally and P. L. Geissler, J. Am. Chem. Soc., 2007, 129, 13847-13856.

31 S. Park and M. D. Fayer, Proc. Natl. Acad. Sci. U. S. A., 2007, 104, 16731-16738. 
32 D. A. Turton, J. Hunger, G. Hefter, R. Buchner and K. Wynne, J. Chem. Phys., 2008, 128, 161102.

33 D. E. Moilanen, D. Wong, D. E. Rosenfeld, E. E. Fenn and M. D. Fayer, Proc. Natl. Acad. Sci. U. S. A., 2009, 106, 375-380.

34 W. Wachter, S. Fernandez, R. Buchner and G. Hefter, J. Phys. Chem. B, 2007, 111, 9010-9017.

35 A. K. Soper and K. Weckstrom, Biophys. Chem., 2006, 124, 180-191.

36 J. Batchelor, A. Olteanu, A. Tripathy and G. Pielak, J. Am. Chem. Soc., 2004, 126, 1958-1961.

37 Y. Marcus, Chem. Rev., 2009, 109, 1346-1370.

38 D. Horinek, A. Herz, L. Vrbka, F. Sedlmeier, S. I. Mamatkulov and R. R. Netz, Chem. Phys. Lett., 2009, 479, 173-183.

39 B. L. Eggimann and J. I. Siepmann, J. Phys. Chem. C, 2008, 112, 210-218.

40 C. D. Wick, J. Chem. Phys., 2008, 131, 084715.

41 D. E. Otten, P. R. Shaffer, P. L. Geissler and R. J. Saykally, Proc. Natl. Acad. Sci. U. S. A., 2012, 109, 701-705.

42 D. H. Powell, A. C. Barnes, J. E. Enderby, G. W. Neilson and P. S. Salmon, Faraday Discuss. Chem. Soc., 1988, 85, 137.

43 H. Ohtaki and T. Radnai, Chem. Rev., 1993, 93, 1157.

44 A. Tongraar, J. T-Thienprasert, S. Rujirawat and S. Limpijumnong, Phys. Chem. Chem. Phys., 2010, 12, 10876.

45 M. Kiriukhin and K. Collins, Biophys. Chem., 2002, 99, 155-168.

46 P. N. Perera, B. Browder and D. Ben-Amotz, J. Phys. Chem. B, 2009, 113, 1805-1809.

47 M. Arshadi, R. Yamdagni and P. Kebarle, J. Phys. Chem., 1970, 74, 1475.

48 K. Hiraoka, S. Mizuse and S. Yamabe, J. Phys. Chem., 1988, 92, 3943.

49 G. Markovich, S. Pollack, R. Giniger and O. Cheshnovsky, J. Chem. Phys., 1994, 101, 9344.

50 M. F. Kropman and H. J. Bakker, Science, 2001, 291, 2118.

51 M. Kropman, H. Nienhuys and H. Bakker, Phys. Rev. Lett., 2002, 88, 077601.

52 H. Bakker, M. Kropman and A. Omta, J. Phys.: Condens. Matter, 2005, 17, S3215-S3224.

53 D. A. Turton, J. Hunger, G. Hefter, R. Buchner and K. Wynne, J. Chem. Phys., 2008, 128, 161102.

54 P. Åke Bergström, J. Lindgren and O. Kristiansson, J. Phys. Chem., 1991, 95, 8575.

55 M. Patra and M. Karttunen, J. Comput. Chem., 2004, 25, 678-689.

56 R. W. Impey, P. A. Madden and I. R. McDonald, J. Phys. Chem., 1983, 87, 5071.

57 A. Chandra, Phys. Rev. Lett., 2000, 85, 768.

58 L. X. Dang, J. E. Rice, J. Caldwell and P. A. Kollman, J. Am. Chem. Soc., 1991, 113, 2481.

59 W. L. Jorgensen and D. L. Severance, J. Chem. Phys., 1993, 99, 4233.

60 D. E. Smith and L. X. Dang, J. Chem. Phys., 1994, 100, 3757. 61 S. J. Stuart and B. J. Berne, J. Phys. Chem., 1996, 100, 11934.
62 I.-C. Yeh, L. Perera and M. L. Berkowitz, Chem. Phys. Lett., 1997, 264, 31.

63 L. X. Dang and D. E. Smith, J. Chem. Phys., 1993, 99, 6950.

64 L. Perera and M. L. Berkowitz, J. Chem. Phys., 1996, 96, 8288.

65 D. J. Tobias, P. Jungwirth and M. Parrinello, J. Chem. Phys, 2001, 114, 7036.

66 J. L. Fulton, G. K. Schenter, M. D. Baer, C. J. Mundy, L. X. Dang and M. Balasubramanian, J. Phys. Chem. B, 2010, 114, 12926-12937.

67 J. M. Heuft and E. J. Meijer, J. Chem. Phys., 2003, 119, 11788.

68 E. Guardia, I. Skarmoutsos and M. Masia, J. Chem. Theory Comput., 2009, 5, 1449.

69 B. S. Mallik and A. Chandra, J. Chem. Phys., 2011, 387, 48-55.

70 C. Krekeler, B. Hess and L. D. Site, J. Chem. Phys., 2006, 125, 054305.

71 A. Tongraar and B. M. Rode, Phys. Chem. Chem. Phys., 2003, 5, 357.

72 J. Chandrasekhar, D. C. Spellmayer and W. L. Jorgensen, J. Am. Chem. Soc., 1984, 106, 903.

73 E. Clementi, R. Barsotti, J. Fromm and R. O. Watts, Theor. Chim. Acta, 1976, 43, 101.

74 K. Heinzinger, Pure Appl. Chem., 1985, 57, 1031.

75 J. A. N. F. G. A. Ignaczak and M. N. D. S. Cordeiro, Electrochim. Acta, 1999, 45, 659.

76 S. Koneshan, J. C. Rasaiah, R. M. Lynden-Bell and S. H. Lee, J. Phys. Chem. B, 1998, 102, 4193-4204.

77 M. Mezei and D. L. Beveridge, J. Phys. Chem., 1981, 74, 6902.

78 P. Hunt, M. Sprik and R. Vuilleumier, Chem. Phys. Lett., 2003, 376, 68.

79 R. Car and M. Parrinello, Phys. Rev. Lett., 1985, 55, 2471.

80 J. Hutter, et al., CPMD 3.13.2, IBM Research Division and MPI Festkoerperforschung Stuttgart, 2008.

81 P. L. Silvestrelli and M. Parrinello, J. Chem. Phys., 1999, 111, 3572.

82 M. Sprik, J. Hutter and M. Parrinello, J. Chem. Phys., 1996, 105(3), 1142.

83 I.-F. W. Kuo, C. J. Mundy, M. J. McGrath, J. I. Siepmann, J. VandeVondele, M. Sprik, J. Hutter, B. Chen, M. L. Klein, F. Mohamed, M. Krack and M. Parrinello, J. Phys. Chem. B, 2004, 108, 12990.

84 A. D. Becke, Phys. Rev. A: At., Mol., Opt. Phys., 1988, 38, 3098-3100.

85 C. Lee, W. Yang and R. G. Parr, Phys. Rev. B: Condens. Matter Mater. Phys., 1988, 37, 785-789.

86 H. J. C. Berendsen, J. R. Grigera and T. P. Straatsma, J. Phys. Chem., 1987, 91, 6269.

87 W. G. Hoover, Phys. Rev. A: At., Mol., Opt. Phys., 1985, 31, 1695.

88 B. Winter and M. Faubel, Chem. Rev., 2006, 106, 1176.

89 P. Hunt and M. Sprik, ChemPhysChem, 2005, 6, 1805-1808.

90 J. Cheng and M. Sprik, Phys. Chem. Chem. Phys., 2012, 14, 11245-11267. 
91 A. Alkauskas, P. Broqvist and A. Pasquarello, Phys. Status Solidi B, 2011, 248, 775-789.

92 C. Adriaanse, J. Cheng, V. Chau, M. Sulpizi, J. VandeVondele and M. Sprik, J. Phys. Chem. Lett., 2012, 3, 3411-3415.

93 M. J. Frisch, G. W. Trucks, H. B. Schlegel, G. E. Scuseria, M. A. Robb, J. R. Cheeseman, G. Scalmani, V. Barone, B. Mennucci, G. A. Petersson, H. Nakatsuji, M. Caricato, X. Li, H. P. Hratchian, A. F. Izmaylov, J. Bloino, G. Zheng, J. L. Sonnenberg, M. Hada, M. Ehara, K. Toyota, R. Fukuda, J. Hasegawa, M. Ishida, T. Nakajima, Y. Honda, O. Kitao, H. Nakai, T. Vreven, J. A. Montgomery, J. E. Peralta, F. Ogliaro, M. Bearpark, J. J. Heyd, E. Brothers, K. N. Kudin, V. N. Staroverov, R. Kobayashi, J. Normand, K. Raghavachari, A. Rendell, J. C. Burant, S. S. Iyengar, J. Tomasi, M. Cossi, N. Rega, J. M. Millam, M. Klene, J. E. Knox, J. B. Cross, V. Bakken, C. Adamo, J. Jaramillo, R. Gomperts, R. E. Stratmann, O. Yazyev, A. J. Austin, R. Cammi, C. Pomelli, J. W. Ochterski, R. L. Martin, K. Morokuma, V. G. Zakrzewski, G. A. Voth, P. Salvador, J. J. Dannenberg, S. Dapprich, A. D. Daniels, O. Farkas, J. B. Foresman, J. V. Ortiz, J. Cioslowski and D. J. Fox, Gaussian 09, Revision A.02, Gaussian, Inc, Wallingford CT, 2009.

94 D. E. Woon and T. H. Dunning, J. Chem. Phys., 1994, 100(4), 2975.

95 H.-C. Chang, J.-C. Jiang, J.-C. Su, C.-Y. Chang and S. H. Lin, J. Phys. Chem. A, 2007, 111, 9201-9206.

96 A. Soper and M. Phillips, Chem. Phys., 1986, 107, 47-60.

97 B. Winter, R. Weber, I. V. Hertel, M. Faubel, P. Jungwirth, E. C. Brown and S. E. Bradforth, J. Am. Chem. Soc., 2005, 127, 7203-7214.

98 R. Seidel, S. Thuiřmer and B. Winter, J. Phys. Chem. Lett., 2011, 2, 633-641.

99 I.-C. Lin, A. P. Seitsonen, I. Tavernelli and U. Rothlisberger, J. Chem. Theory Comput., 2012, 8, 3902-3910.
100 Z. Ma, Y. Zhang and M. E. Tuckerman, J. Chem. Phys., 2012, 137, 044506.

101 R. Richert, J. Phys.: Condens. Matter, 2002, 14, R703.

102 H. Frauenfelder, G. Chen, J. Berendzen, P. W. Fenimore, H. Jansson, B. H. McMahon, I. R. Stroe, J. Swenson and R. D. Young, Proc. Natl. Acad. Sci. U. S. A., 2009, 106, 5129. 103 S. Raugei and M. Klein, J. Chem. Phys., 2002, 116, 196-202. 104 P. L. Silvestrelli, N. Marzari, D. Vanderbilt and M. Parrinello, Solid State Commun., 1998, 107, 7.

105 N. Marzari and D. Vanderbilt, Phys. Rev. B: Condens. Matter Mater. Phys., 1997, 56, 12847.

106 D. Bucher and S. Kuyucak, J. Phys. Chem. B, 2008, 112, 10786.

107 D. Bucher and S. Kuyucak, Chem. Phys. Lett., 2009, 477, 207.

108 J. K. Gregory, D. C. Clary, K. Liu, M. G. Brown and R. J. Saykally, Science, 1997, 275, 814.

109 L. Bernasconi, J. Blumberger, M. Sprik and R. Vuilleumier, J. Chem. Phys., 2004, 121, 11885.

110 C. Terrier, P. Vitorge, M.-P. Gaigeot, R. Spezia and R. Vuilleumier, J. Chem. Phys., 2010, 133, 044509.

111 K. Laasonen, M. Sprik, M. Parrinello and R. Car, J. Chem. Phys., 1993, 99, 9080-9089.

112 Y. S. Badyal, M.-L. Saboungi, D. L. Price, S. D. Shastri, D. R. Haeffner and A. K. Soper, J. Chem. Phys., 2000, 112, 9206-9208.

113 L. Petit, R. Vuilleumier, P. Maldivi and C. Adamo, J. Chem. Theory Comput., 2008, 4, 1040-1048.

114 A. Öhrn and G. Karlström, J. Phys. Chem. B, 2004, 108, 8452-8459.

115 D. Laage and J. T. Hynes, J. Phys. Chem. B, 2008, 112, 14230-14242.

116 D. Laage and J. T. Hynes, Science, 2006, 311, 832-835.

117 D. Laage and J. T. Hynes, Proc. Natl. Acad. Sci. U. S. A., 2007, 104, 11167-11172. 